\title{
An Efficient and Enantioselective Approach to The Azaspirocyclic Core of Alkaloids: Formal Synthesis of Halichlorine and Pinnaic acid
}

\author{
Hong-Lu Zhang, Gang Zhao*,Yu Ding* ,Bin Wu
}

Laboratory of Modern Organic Synthetic Chemistry, Shanghai Institute of Organic Chemistry, Chinese Academy of Sciences, 354 Fenglin Road, Shanghai 200032, P.R. China.

\section{Content:}

S 2: ${ }^{1} \mathrm{H}$ NMR of compound (+) 6 . $\mathrm{S} 4:{ }^{13} \mathrm{C}$ NMR of compound 8 . S 6: ${ }^{13} \mathrm{C}$ NMR of compound 9. S 8: ${ }^{13} \mathrm{C}$ NMR of compound 10. S 10: ${ }^{13} \mathrm{C}$ NMR of compound 11 . S 12: ${ }^{13} \mathrm{C}$ NMR of compound 12. S 14: ${ }^{13} \mathrm{C}$ NMR of compound 13. S 16: ${ }^{13} \mathrm{C}$ NMR of compound 14. S 18: ${ }^{13} \mathrm{C}$ NMR of compound 15. S 20: ${ }^{13} \mathrm{C}$ NMR of compound 16. S 22: ${ }^{13} \mathrm{C}$ NMR of compound 18. S 24: ${ }^{13} \mathrm{C}$ NMR of compound 19. S 26: ${ }^{13} \mathrm{C}$ NMR of compound 23. S 28: ${ }^{13} \mathrm{C}$ NMR of compound 24. S 30: ${ }^{13} \mathrm{C}$ NMR of compound 25. S 32: ${ }^{13} \mathrm{C}$ NMR of compound 26. S 34: ${ }^{13} \mathrm{C}$ NMR of compound 27. S 36: ${ }^{13} \mathrm{C}$ NMR of compound 28. S 38: ${ }^{13} \mathrm{C}$ NMR of compound 30. $\mathrm{S} 40:{ }^{13} \mathrm{C}$ NMR of compound 31. S 42: ${ }^{13} \mathrm{C}$ NMR of compound 3.
S 3: ${ }^{1} \mathrm{H}$ NMR of compound 8. S 5: ${ }^{1} \mathrm{H}$ NMR of compound 9. S 7: ${ }^{1} \mathrm{H}$ NMR of compound 10. S 9: ${ }^{1} \mathrm{H}$ NMR of compound 11. S 11: ${ }^{1} \mathrm{H}$ NMR of compound 12. S 13: ${ }^{1} \mathrm{H}$ NMR of compound 13. S 15: ${ }^{1} \mathrm{H}$ NMR of compound 14. S 17: ${ }^{1} \mathrm{H}$ NMR of compound 15. S 19: ${ }^{1} \mathrm{H}$ NMR of compound 16. S 21: ${ }^{1} \mathrm{H}$ NMR of compound 18. S 23: ${ }^{1} \mathrm{H}$ NMR of compound 19. S 25: ${ }^{1} \mathrm{H}$ NMR of compound 23. S 27: ${ }^{1} \mathrm{H}$ NMR of compound 24. S 29: ${ }^{1} \mathrm{H}$ NMR of compound 25. S 31: ${ }^{1} \mathrm{H}$ NMR of compound 26. S 33: ${ }^{1} \mathrm{H}$ NMR of compound 27. S 35: ${ }^{1} \mathrm{H}$ NMR of compound 28. S 37: ${ }^{1} \mathrm{H}$ NMR of compound 30. S 39: ${ }^{1} \mathrm{H}$ NMR of compound 31. S 41: ${ }^{1} \mathrm{H}$ NMR of compound 3.

S 43: ORTEP drawing of the X-ray structure of 28

\section{Experimental:}

All reactions were conducted under Ar atmosphere unless stated otherwise and monitored by TLC on precoated silica gel plates. Column chromatography was performed on silica gel 300-400 mesh and eluted with hexane and ethyl acetate mixtures. All solvents were refluxed and distilled from sodium benzophenone ketyl (THF, $\left.\mathrm{Et}_{2} \mathrm{O}\right)$ or $\mathrm{CaH}_{2}\left(\mathrm{CH}_{2} \mathrm{Cl}_{2}\right)$.

The NMR spectra were recorded at $300 \mathrm{MHz}\left({ }^{1} \mathrm{H}\right)$ and $75.0 \mathrm{MHz}\left({ }^{13} \mathrm{C}\right)$, and reported in $\delta$-units (ppm) and $\mathrm{J}$-values $(\mathrm{Hz})$ with $\mathrm{Me}_{4} \mathrm{Si}$ as the internal standard. MS spectra (EI) were recorded. Infrared (IR) spectra were reported in wave numbers $\left(\mathrm{cm}^{-1}\right)$. Optical rotations $[\alpha]_{D}$-values were reported in units of $10^{-1} \mathrm{deg} \mathrm{cm}^{3} \mathrm{~g}^{-1}$. Element analyses were performed in the Analysis Center of Shanghai Institute of Organic Chemistry. 

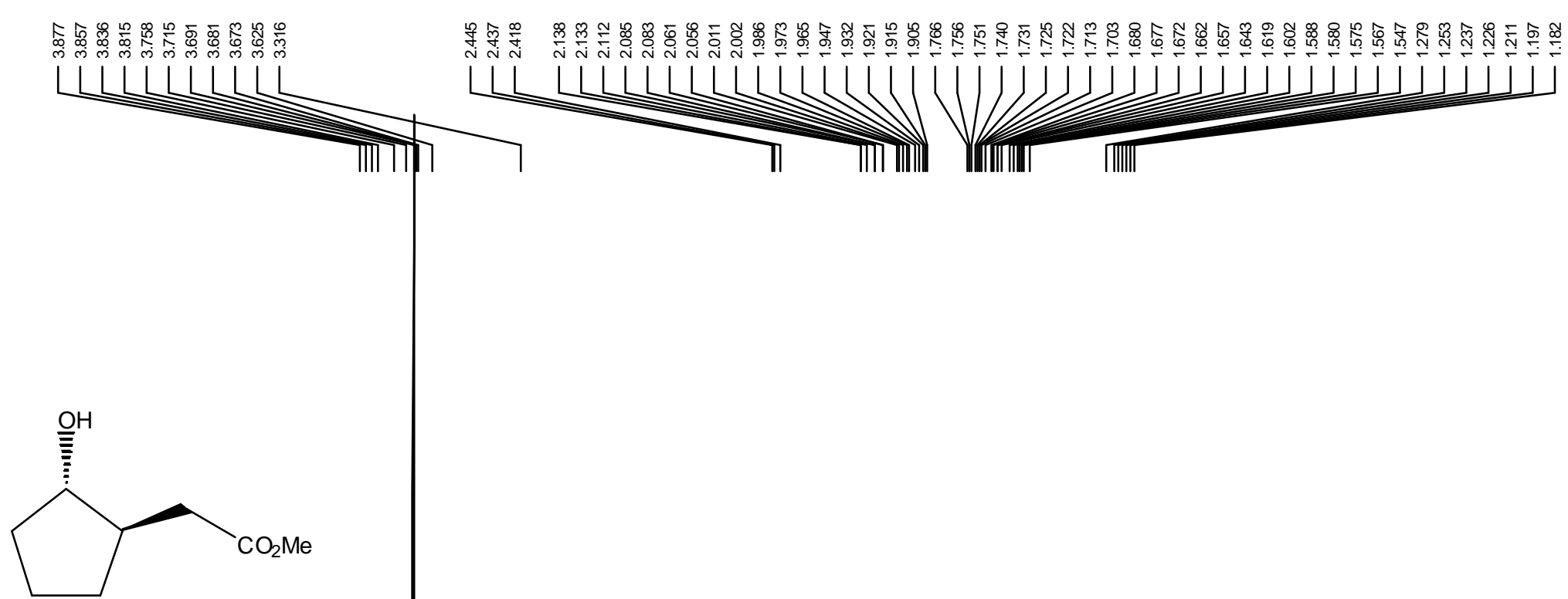

Compound (+) 6

${ }^{1} \mathrm{HNMR}\left(300 \mathrm{MHz}, \mathrm{CDCl}_{3}\right)$

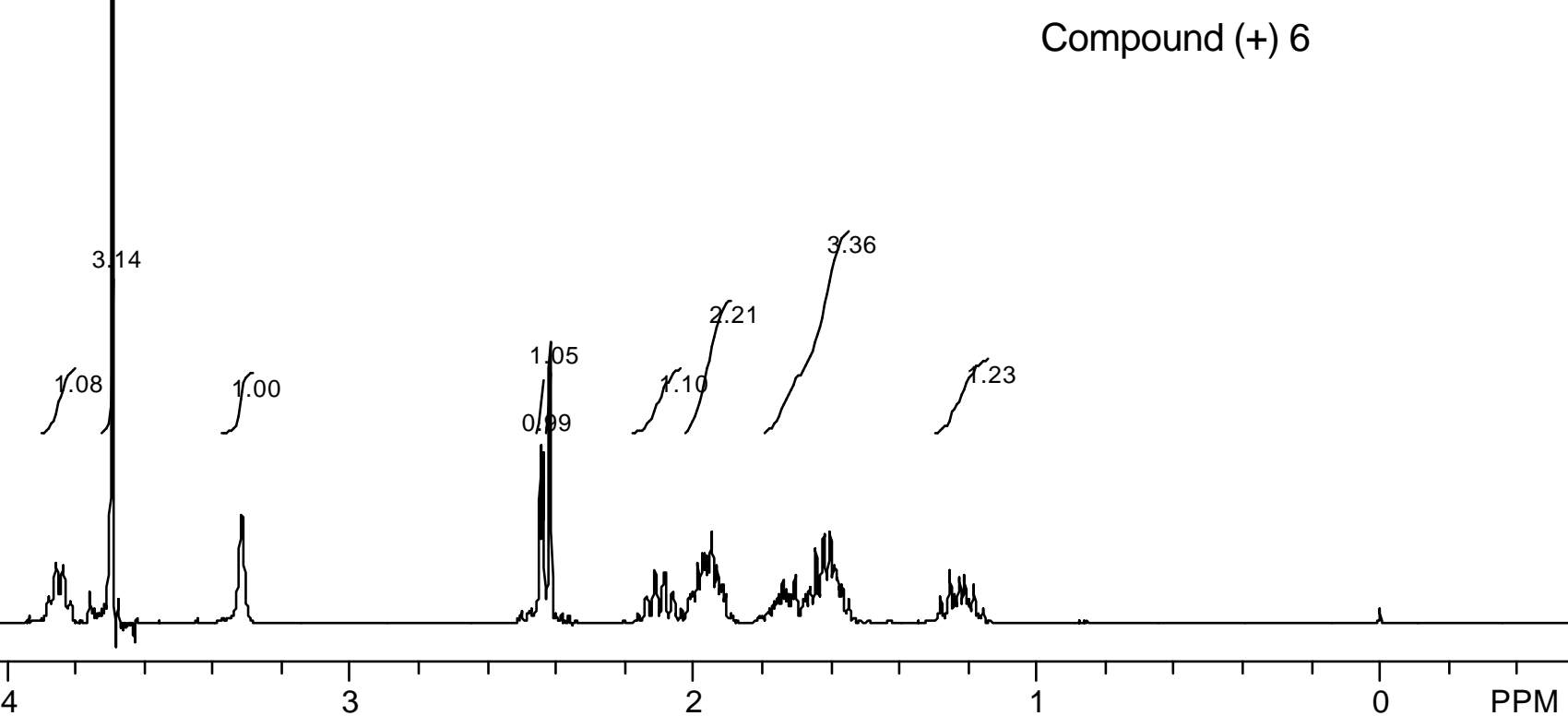

$\mathrm{S} 2$ 

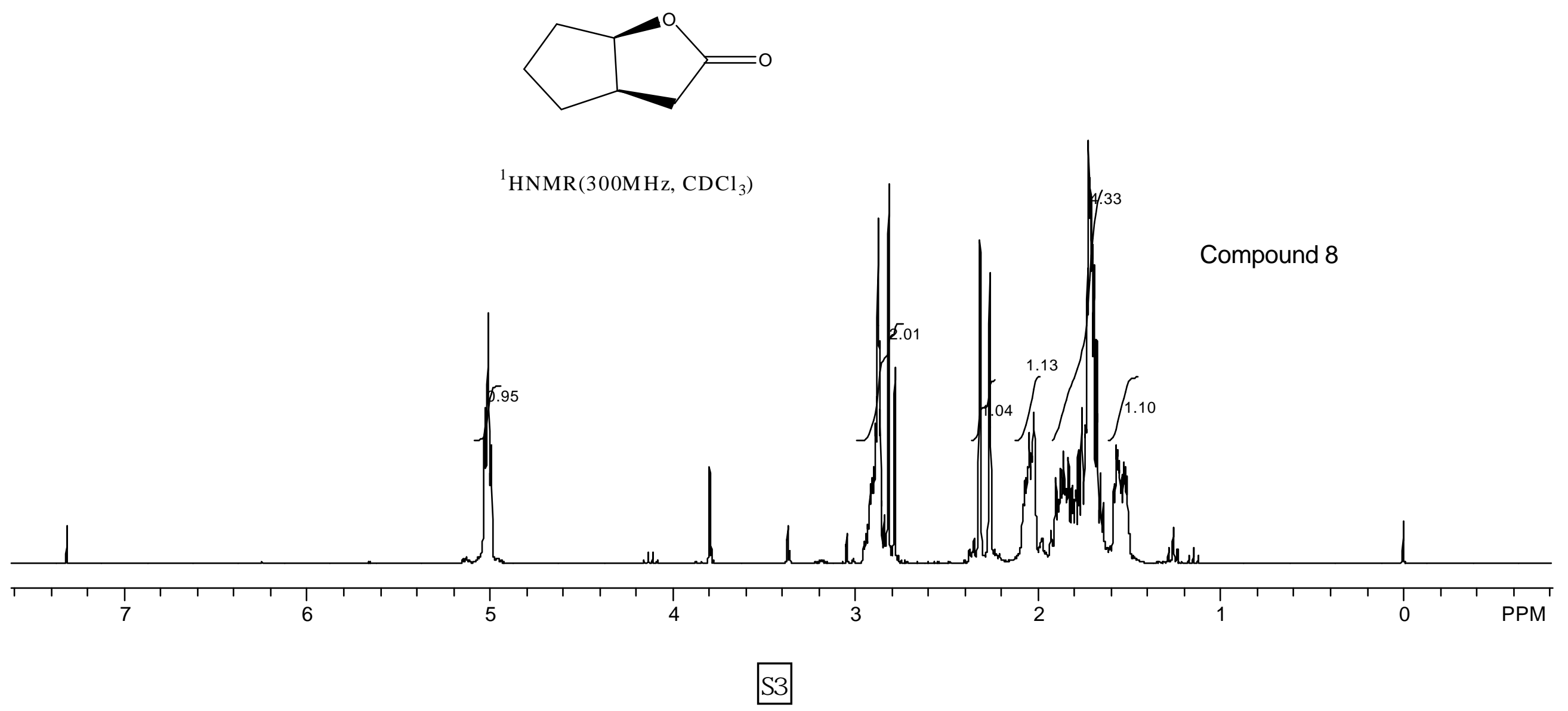

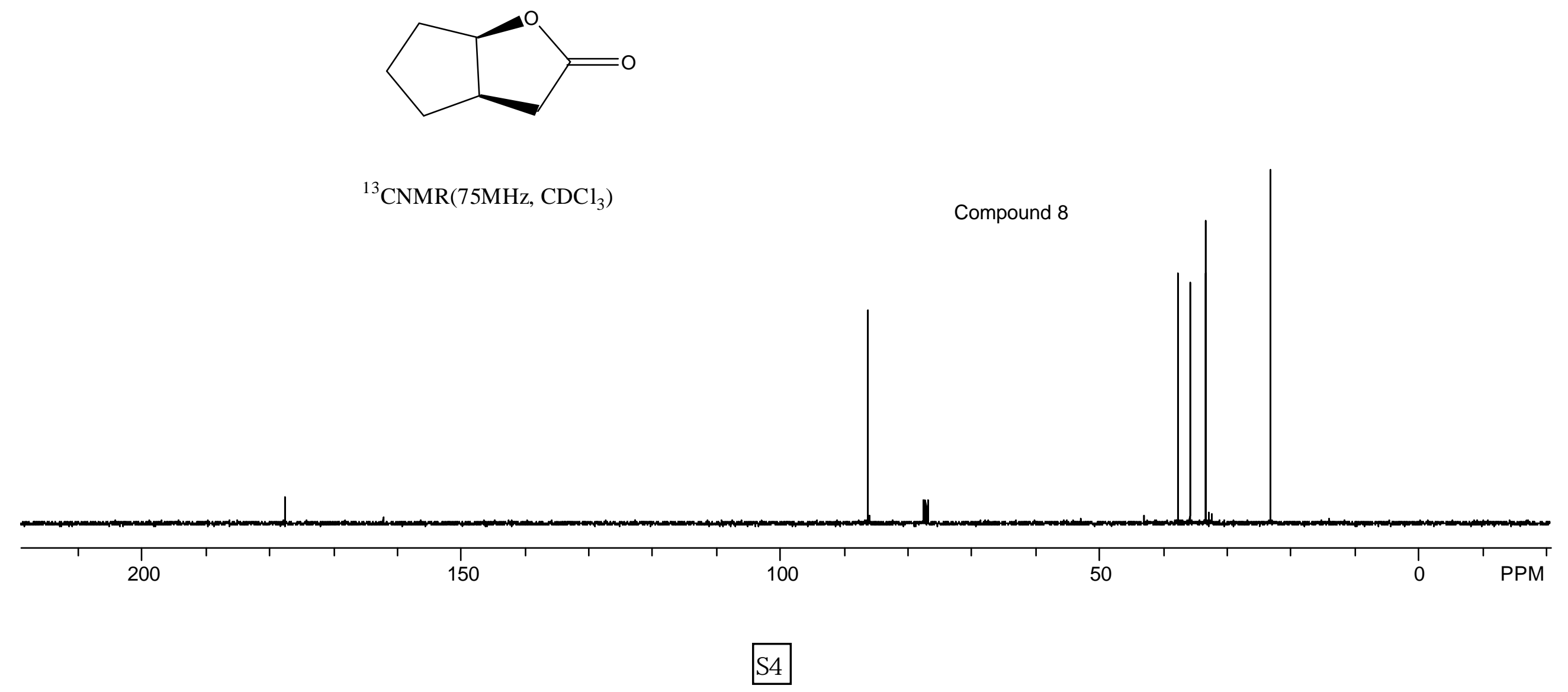


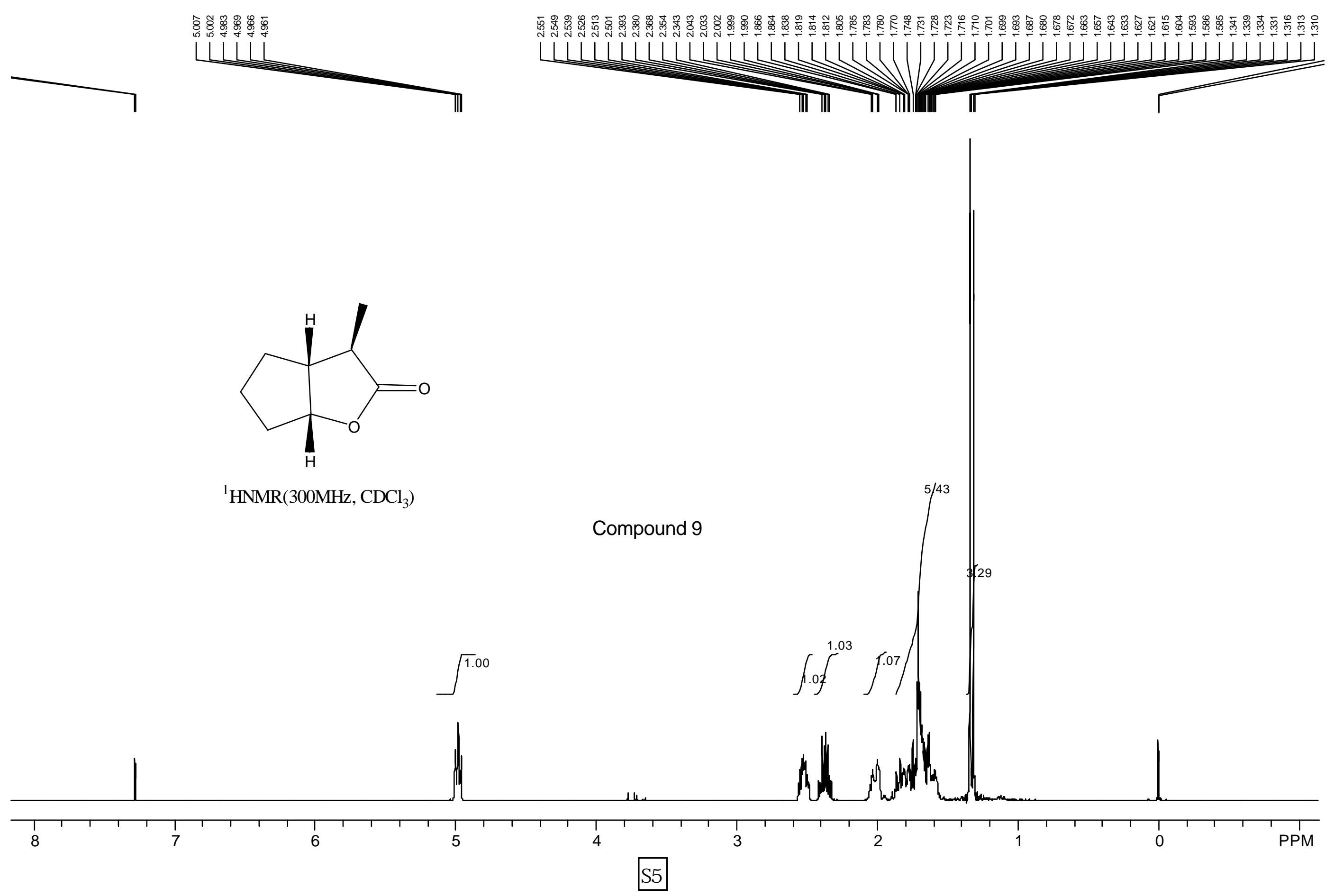




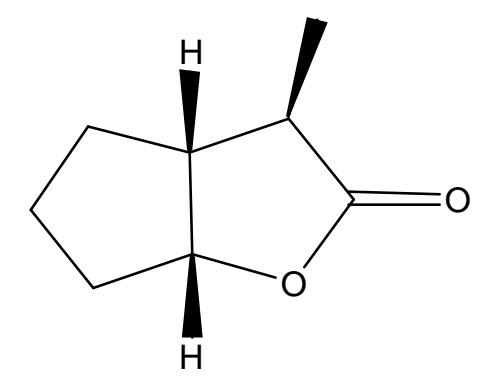

${ }^{13} \mathrm{CNMR}\left(75 \mathrm{MHz}, \mathrm{CDCl}_{3}\right)$

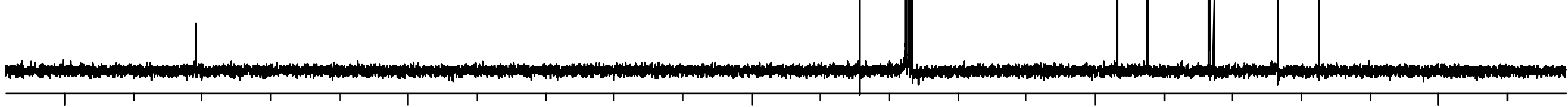



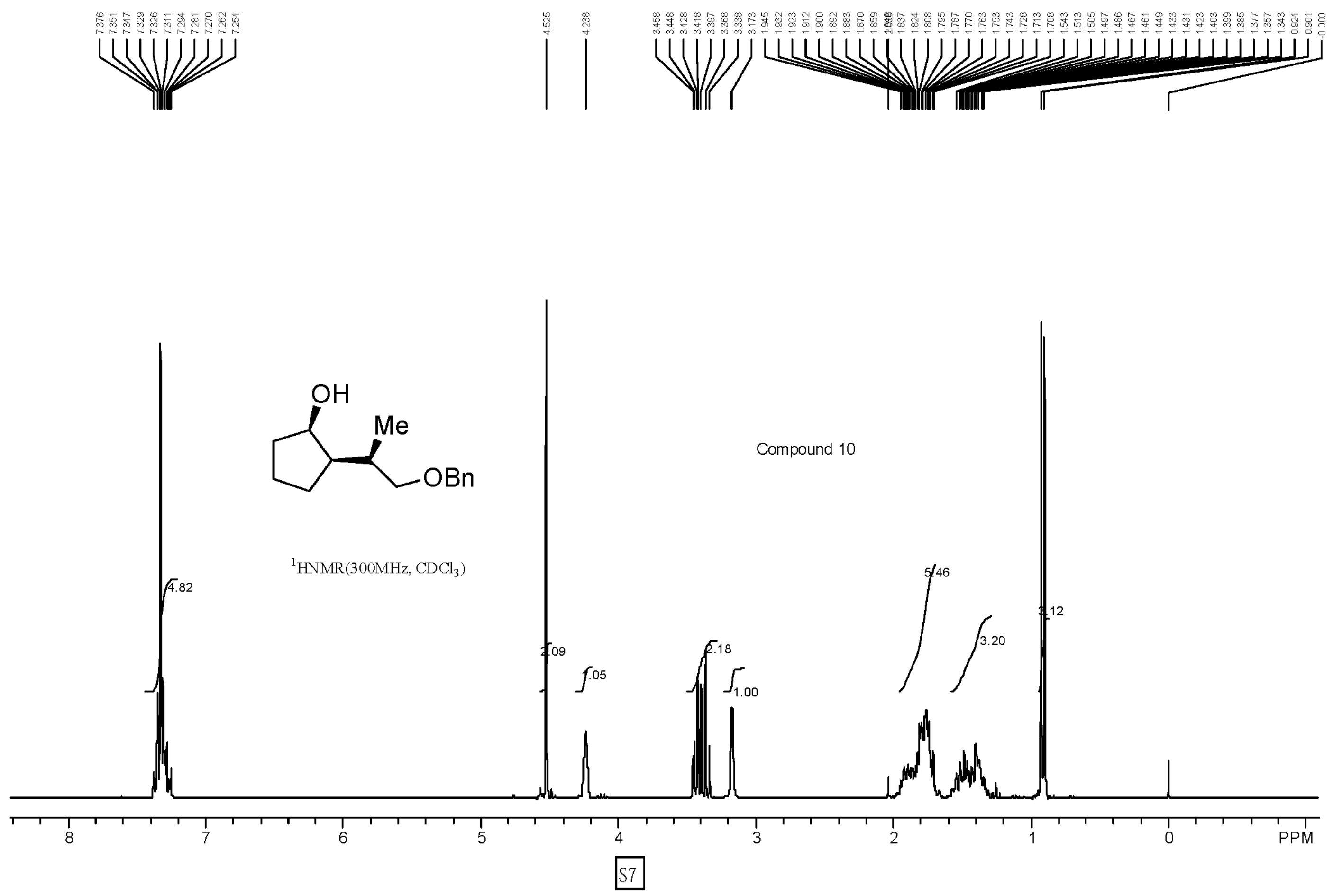


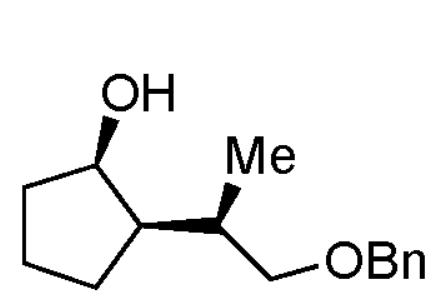

${ }^{13} \mathrm{CNMR}\left(75 \mathrm{MHz}, \mathrm{CDCl}_{3}\right)$

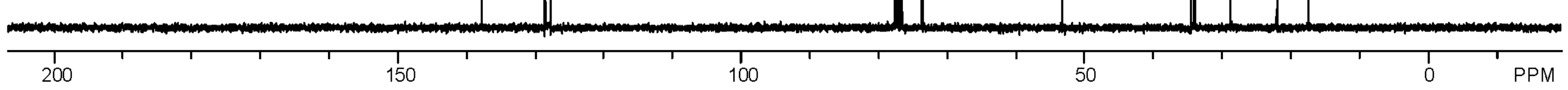



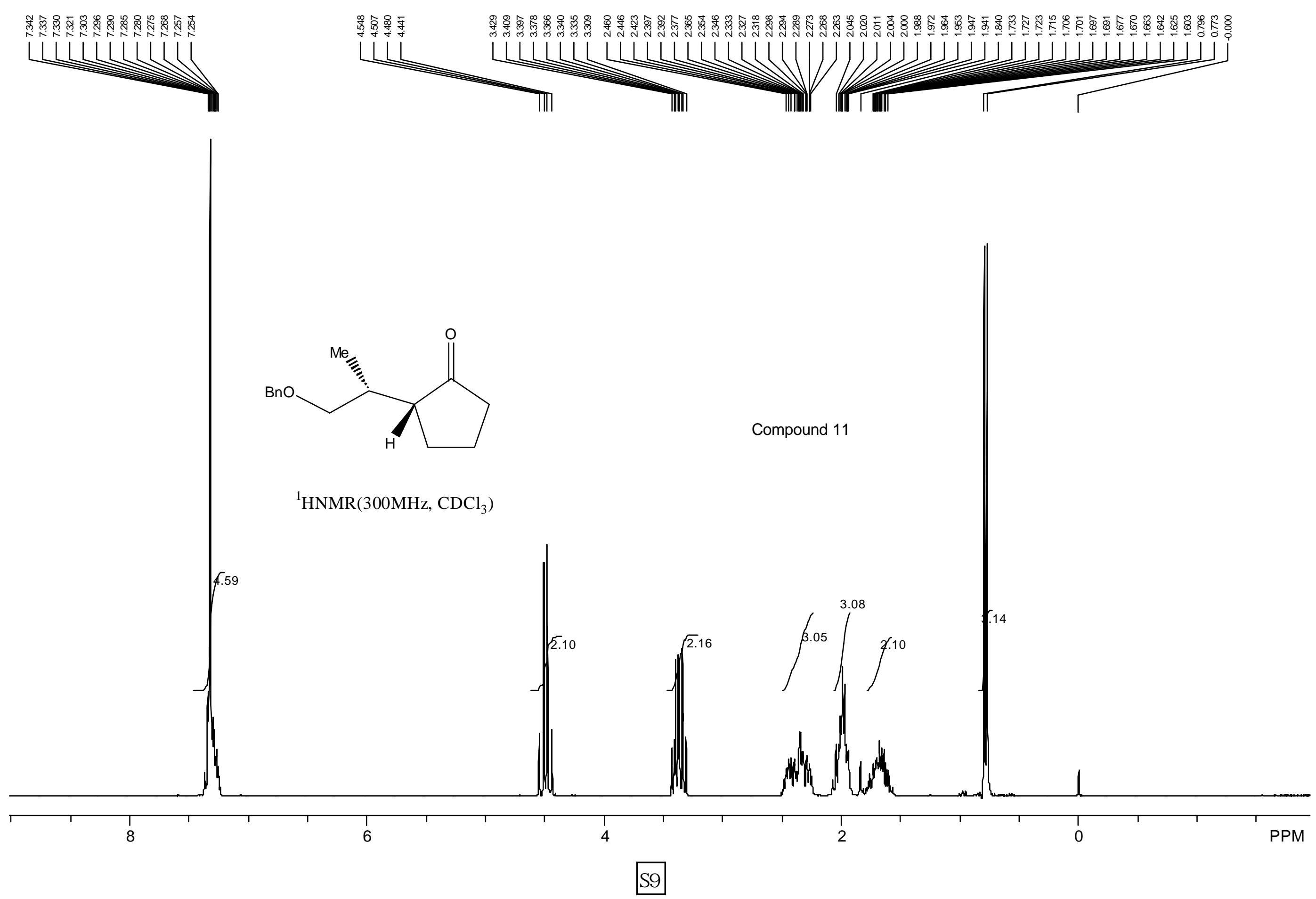


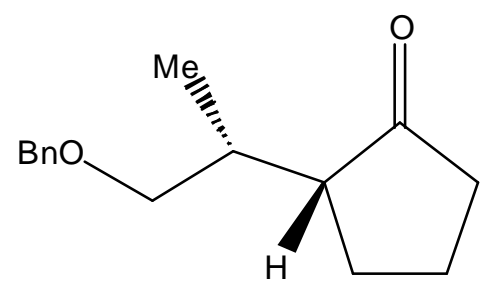

Compound 11

${ }^{13} \mathrm{CNMR}\left(75 \mathrm{MHz}, \mathrm{CDCl}_{3}\right)$

2

250 


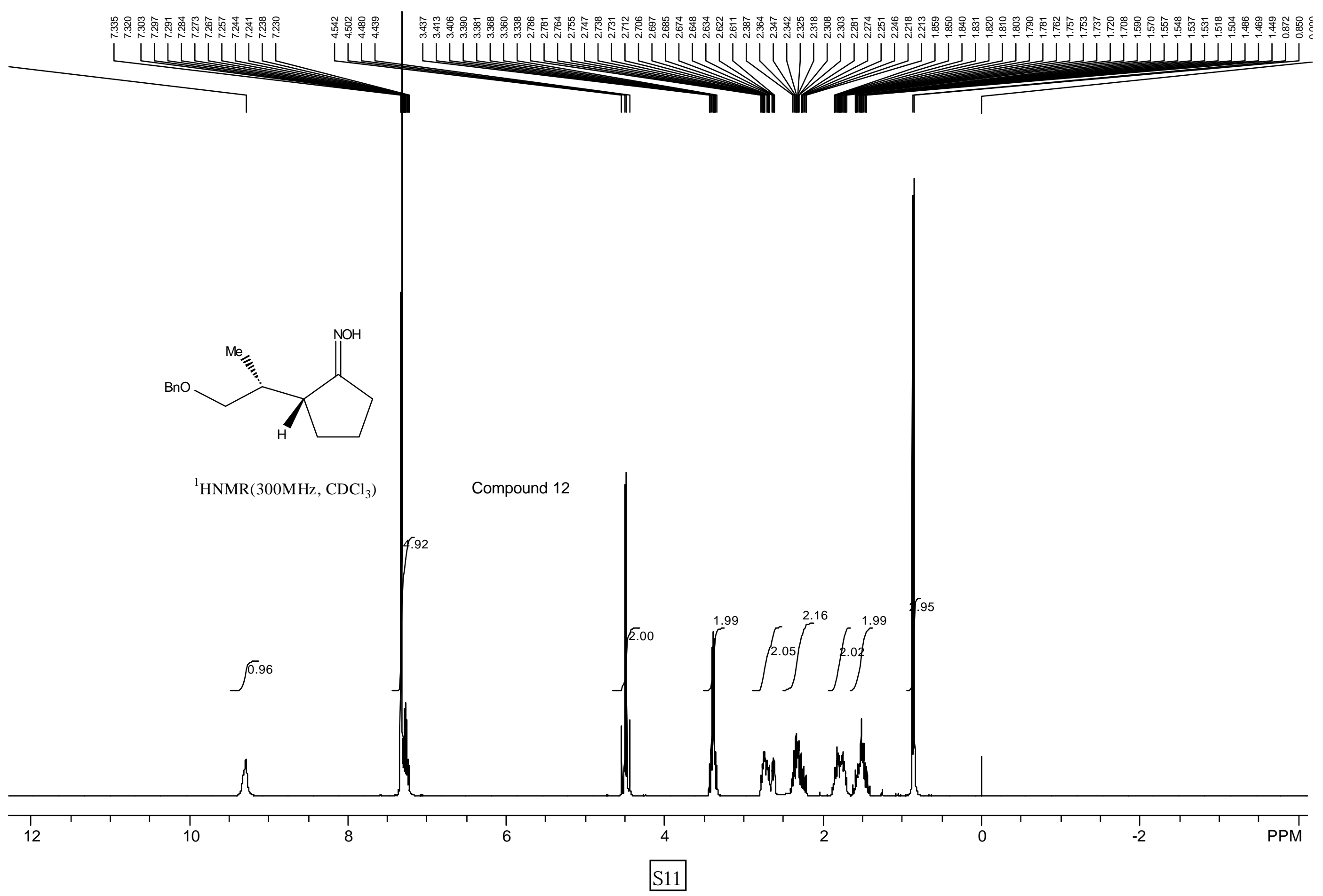




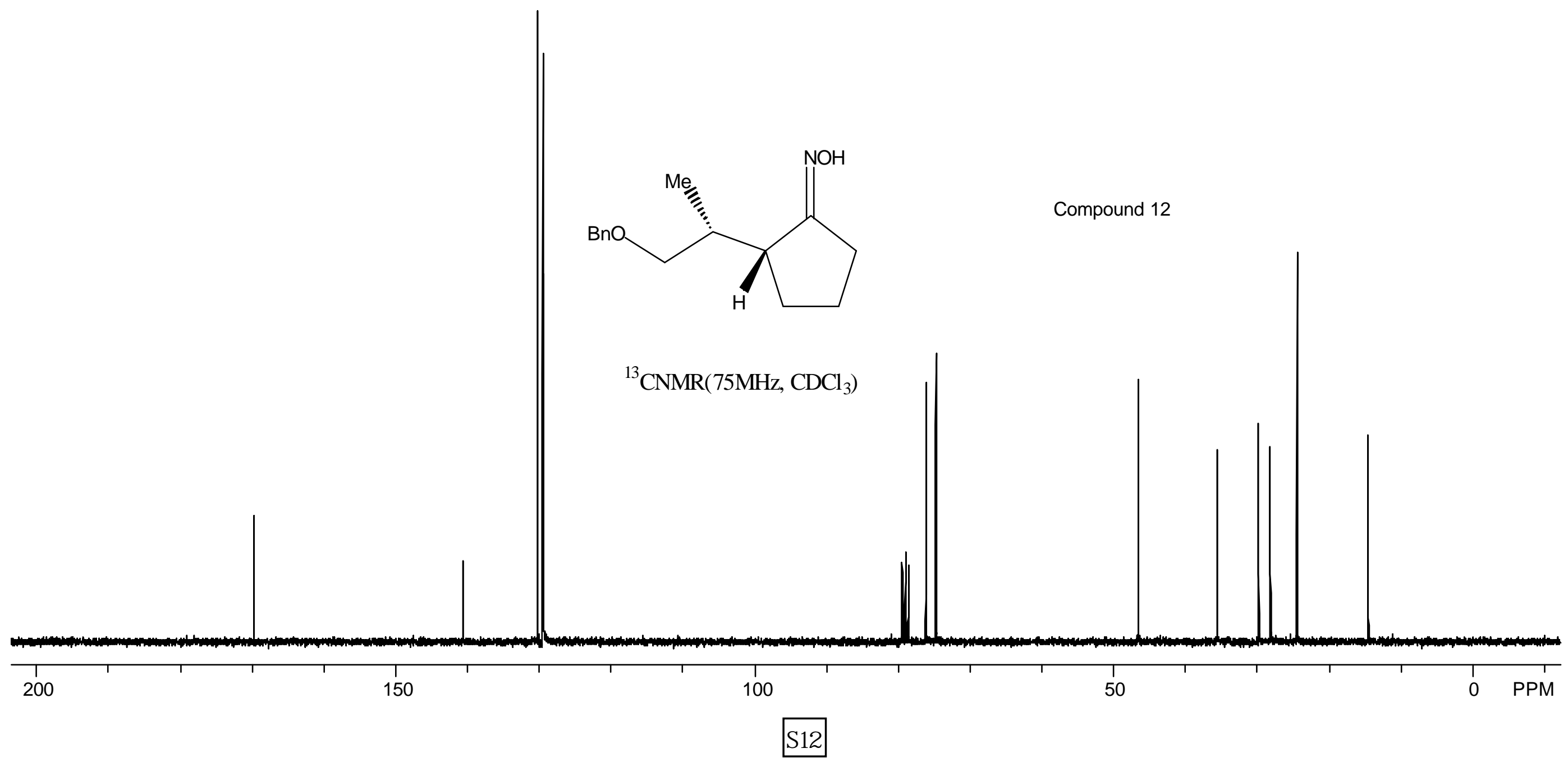



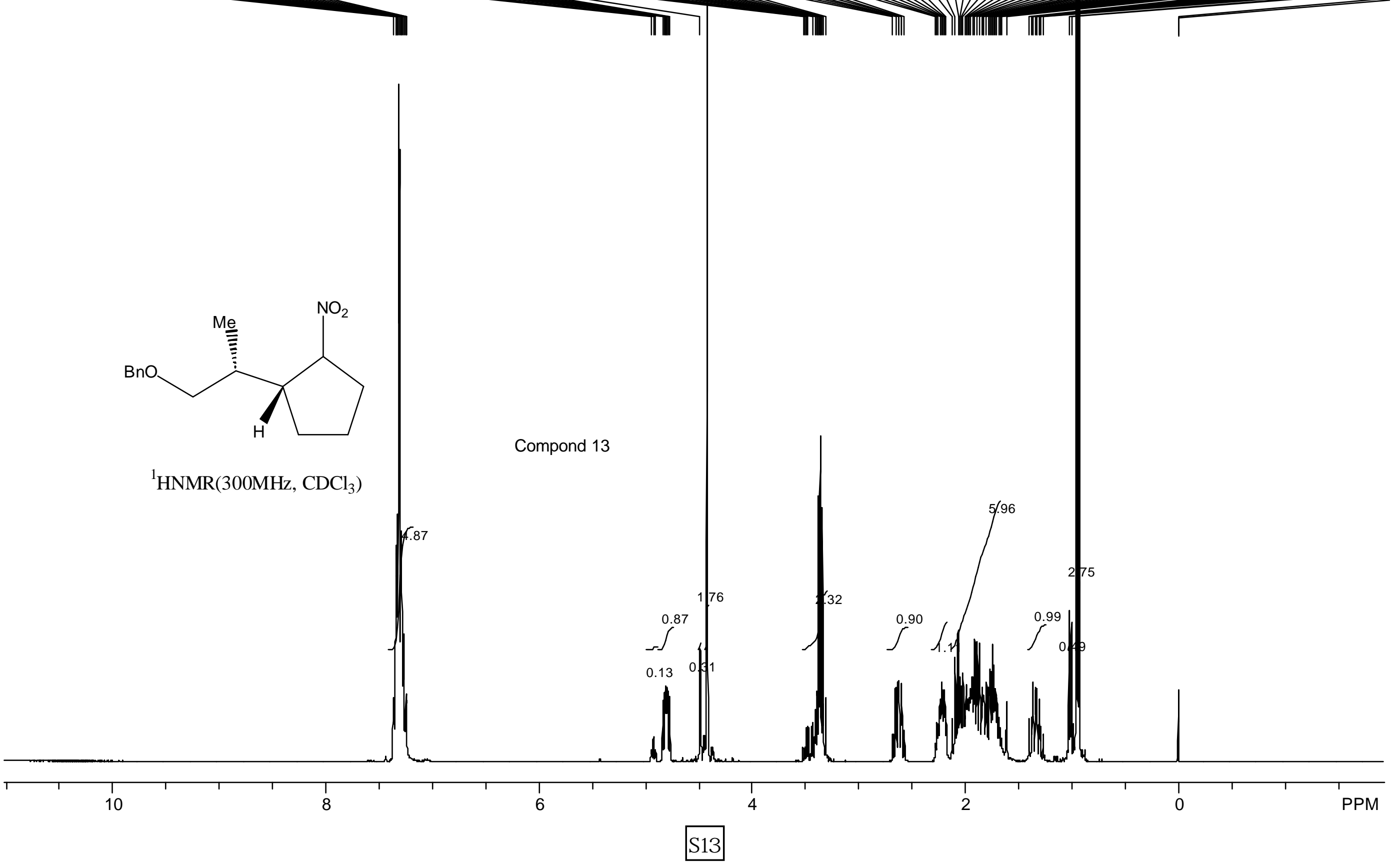


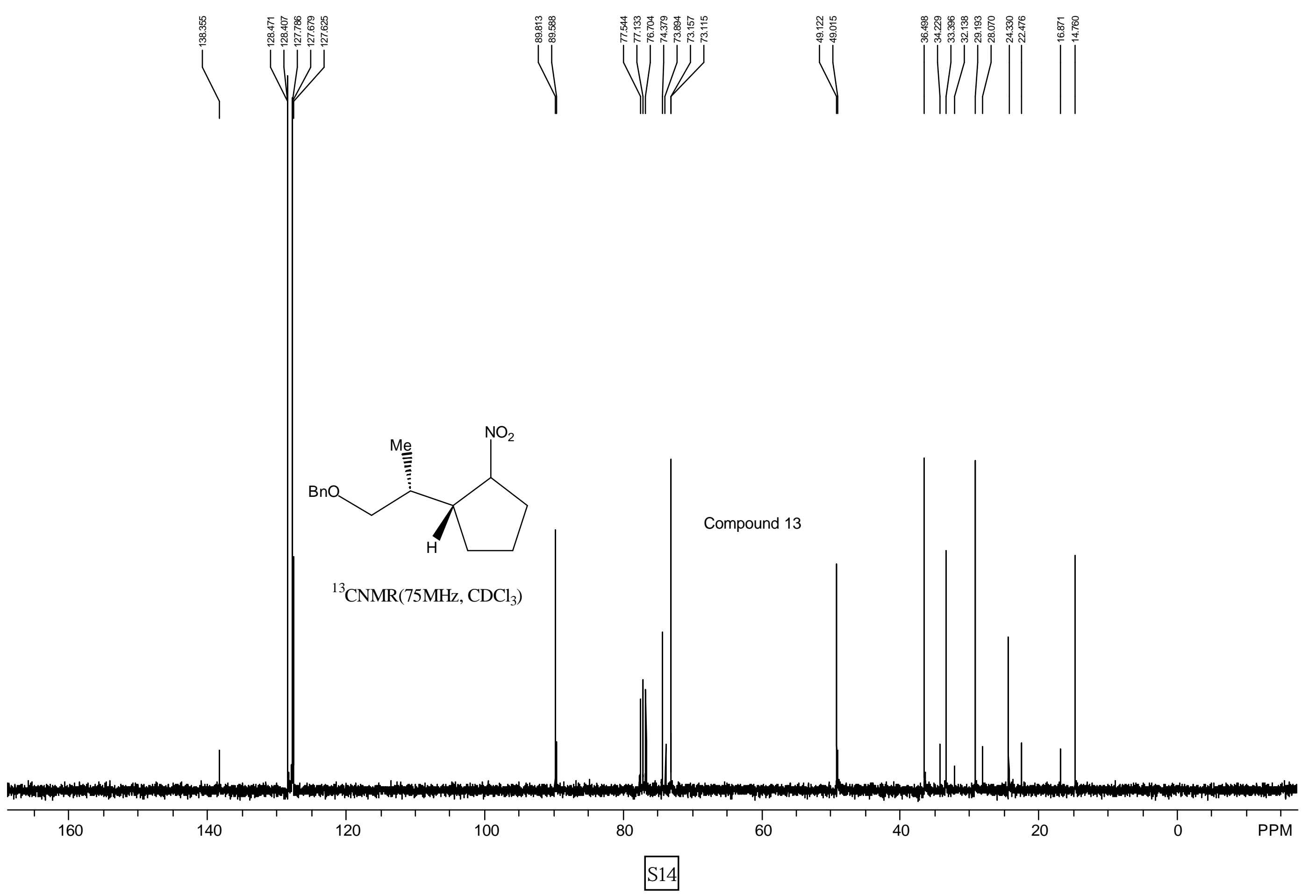




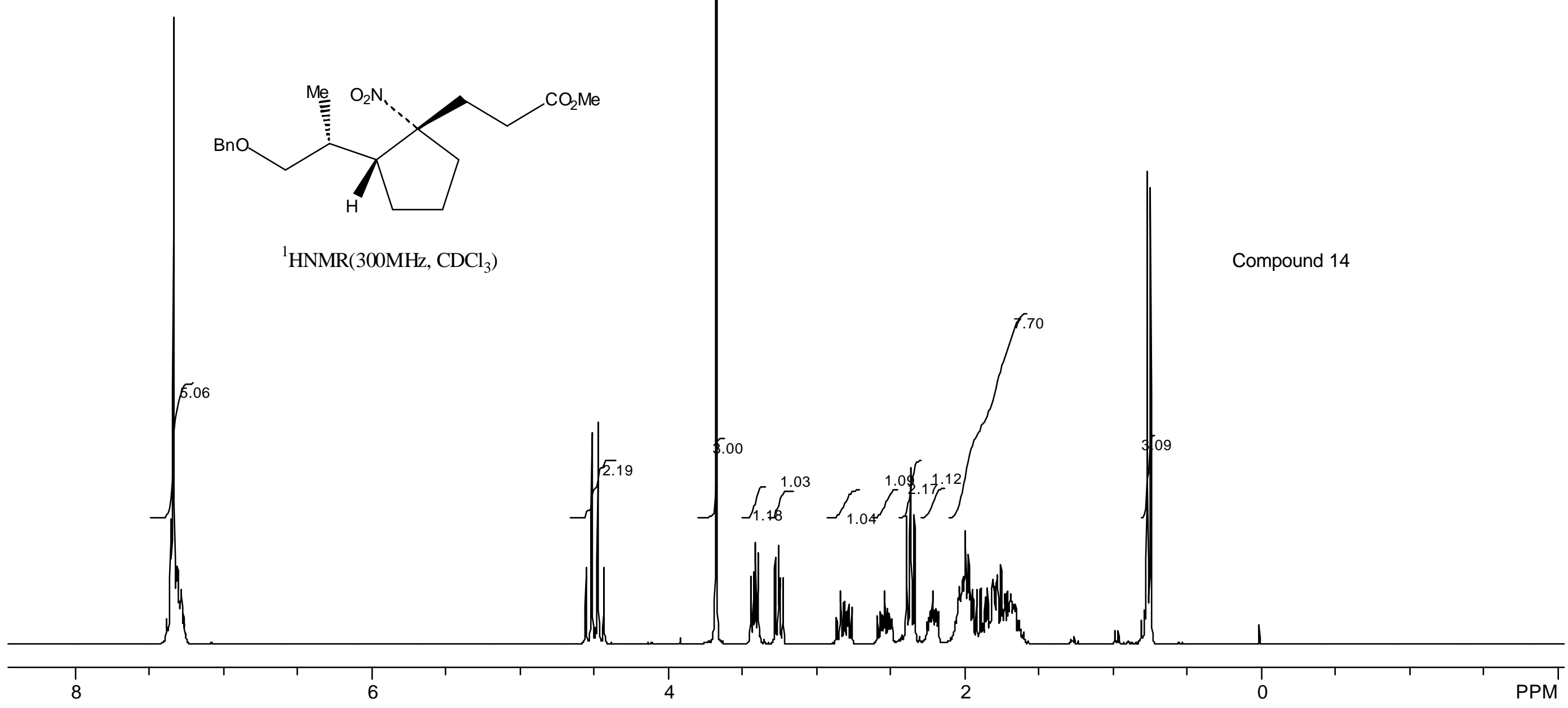

S15 

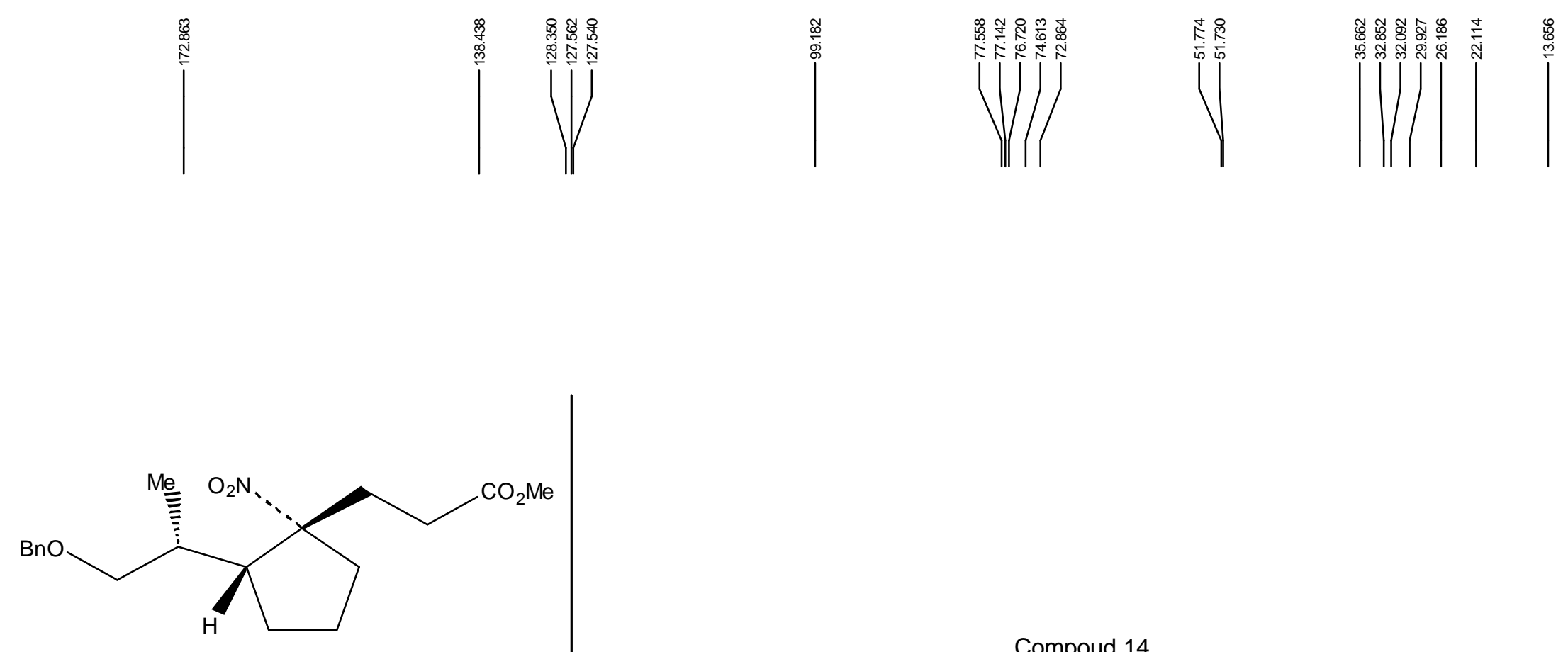

${ }^{13} \mathrm{CNMR}\left(75 \mathrm{MHz}, \mathrm{CDCl}_{3}\right)$

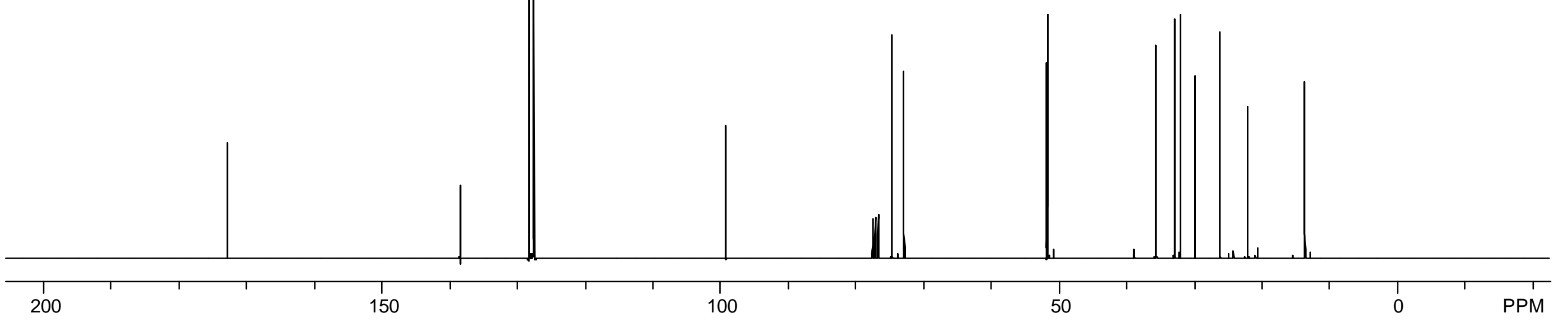



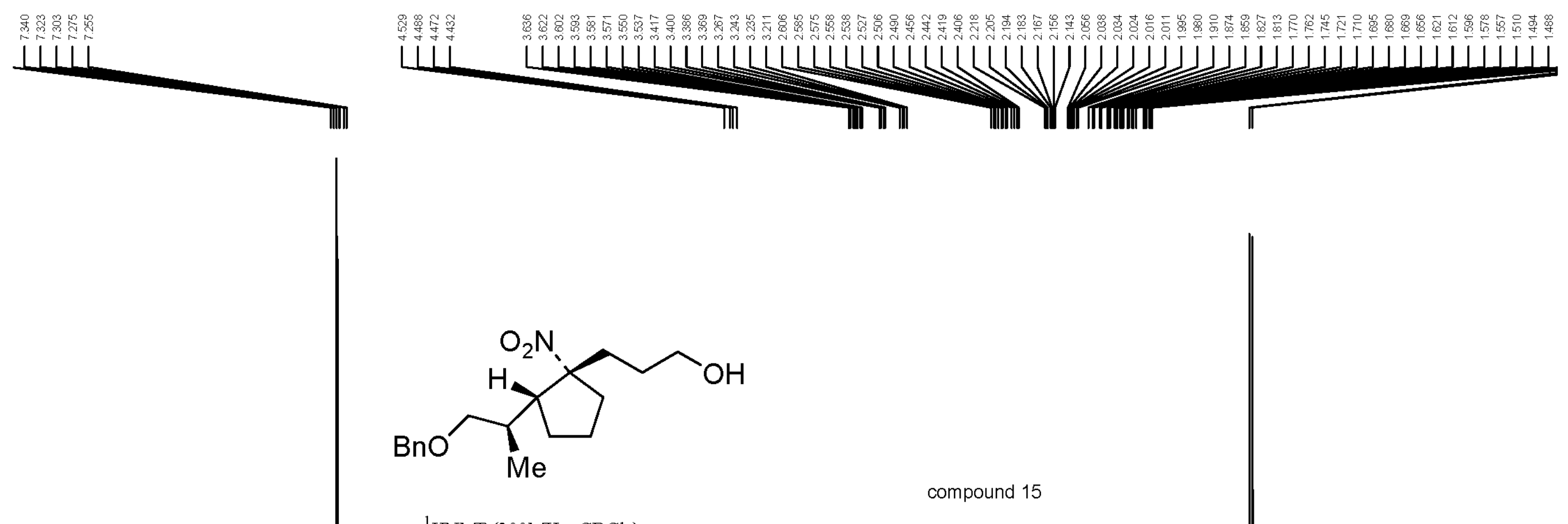

${ }^{1} \mathrm{HNMR}\left(300 \mathrm{MHz}, \mathrm{CDCl}_{3}\right.$ )

compound 15
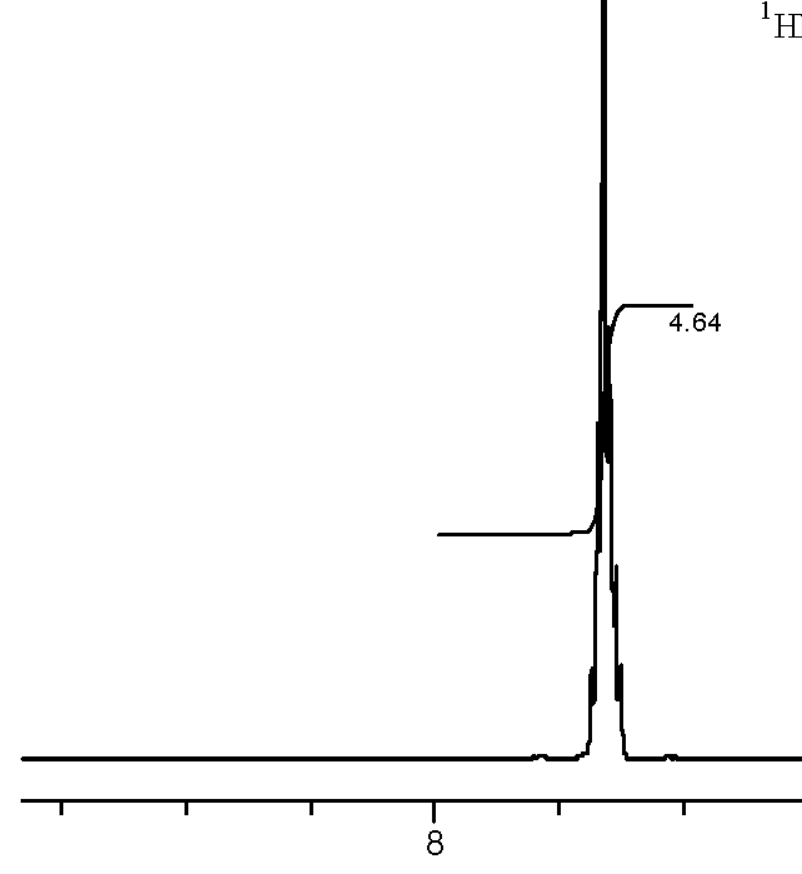


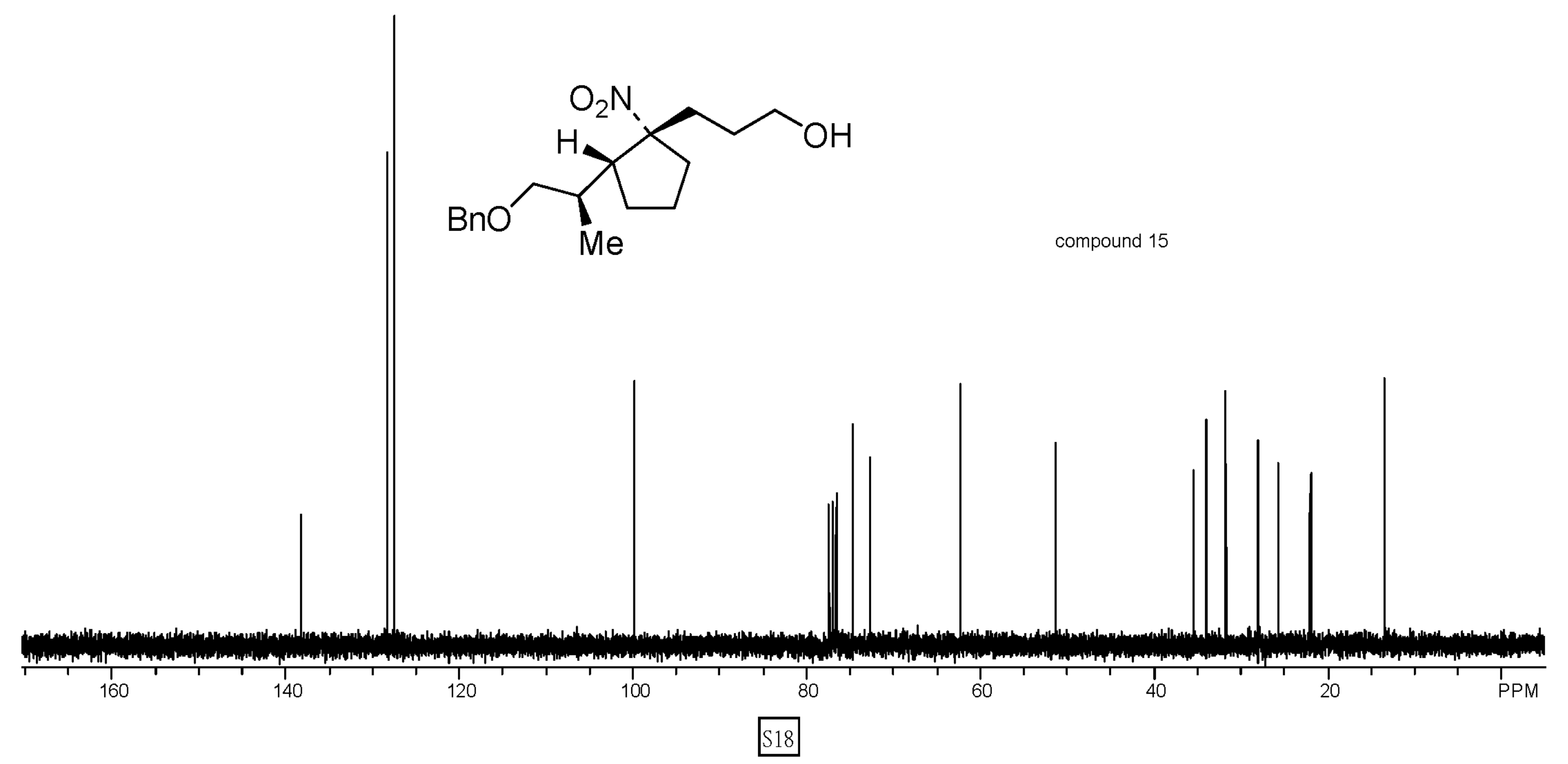


Compound 16

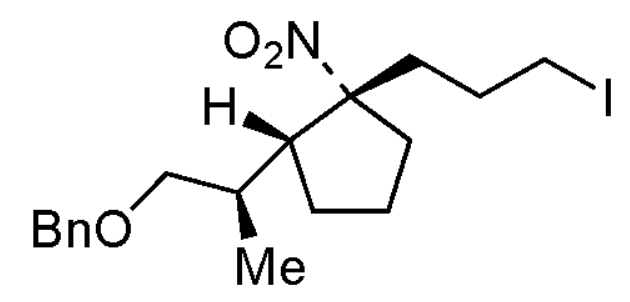

${ }^{1} \mathrm{HNMR}\left(300 \mathrm{MHz}, \mathrm{CDCl}_{3}\right)$

$$
\text { (1) }
$$
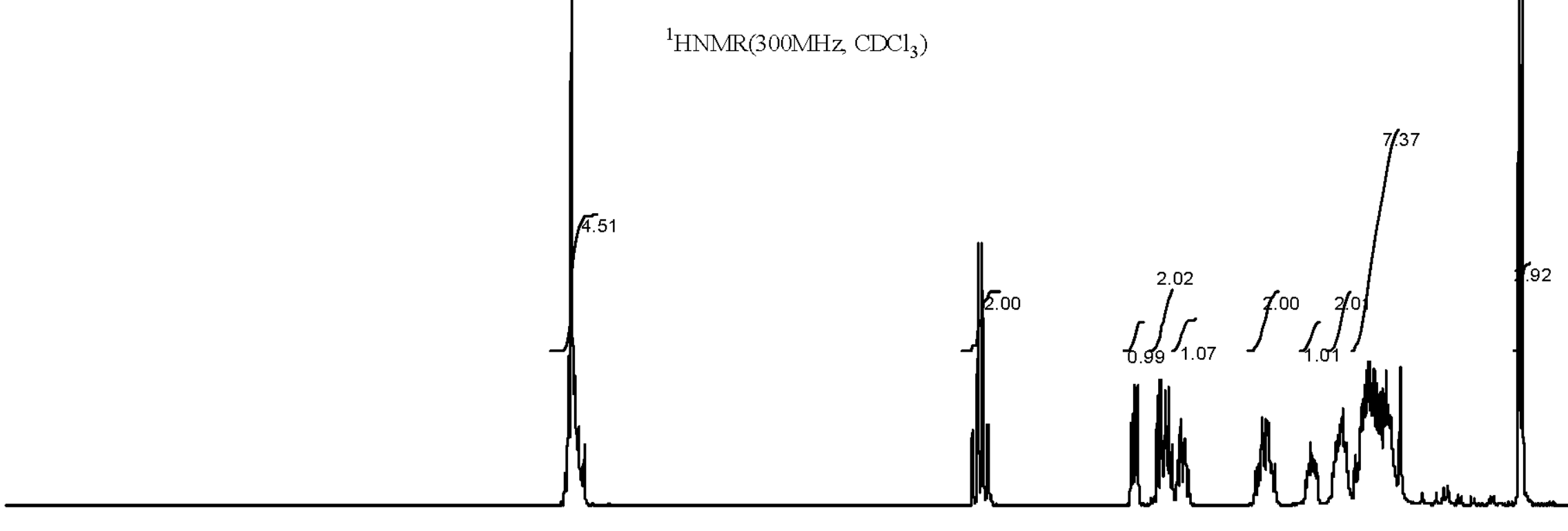

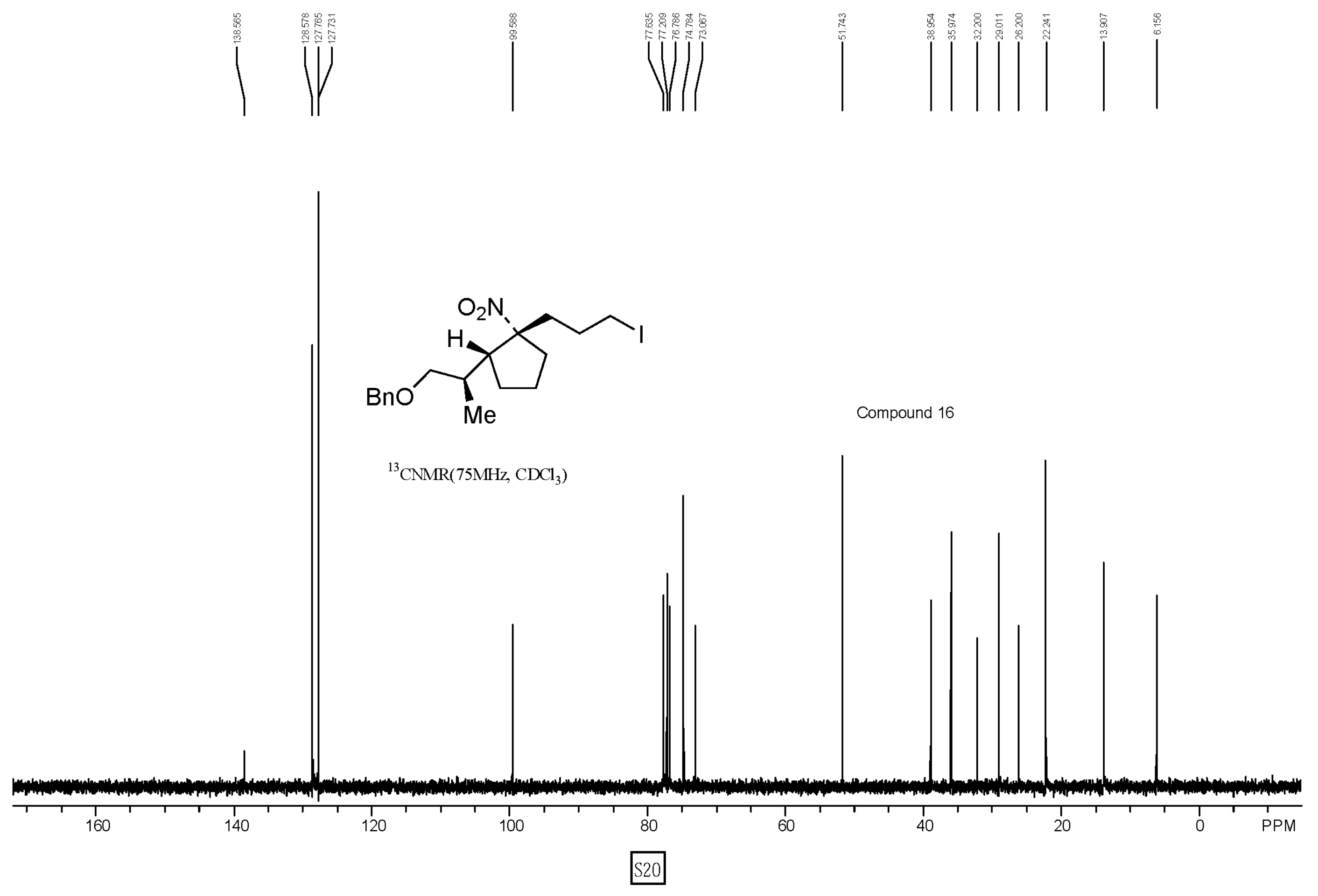


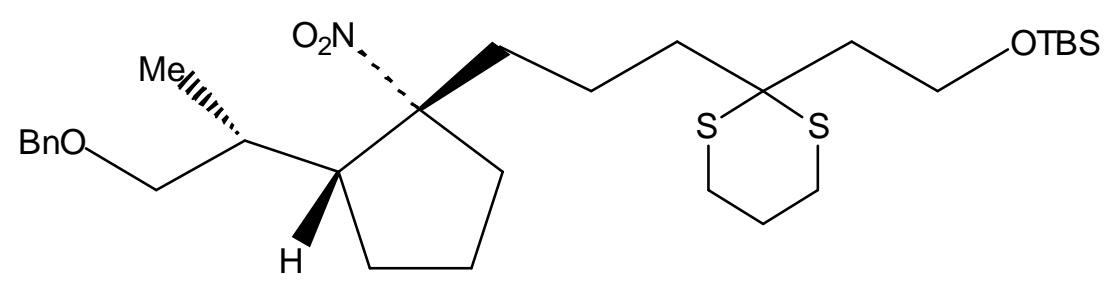

${ }^{1} \mathrm{HNMR}\left(300 \mathrm{MHz}, \mathrm{CDCl}_{3}\right)$

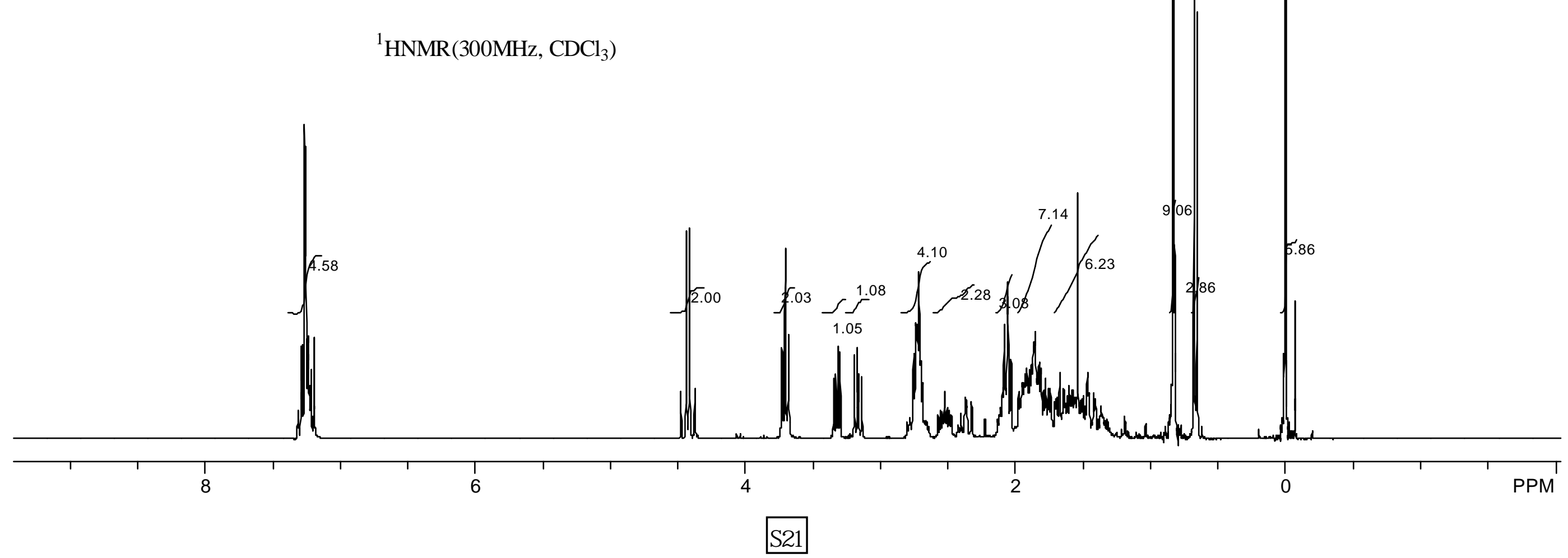

Compound 18 


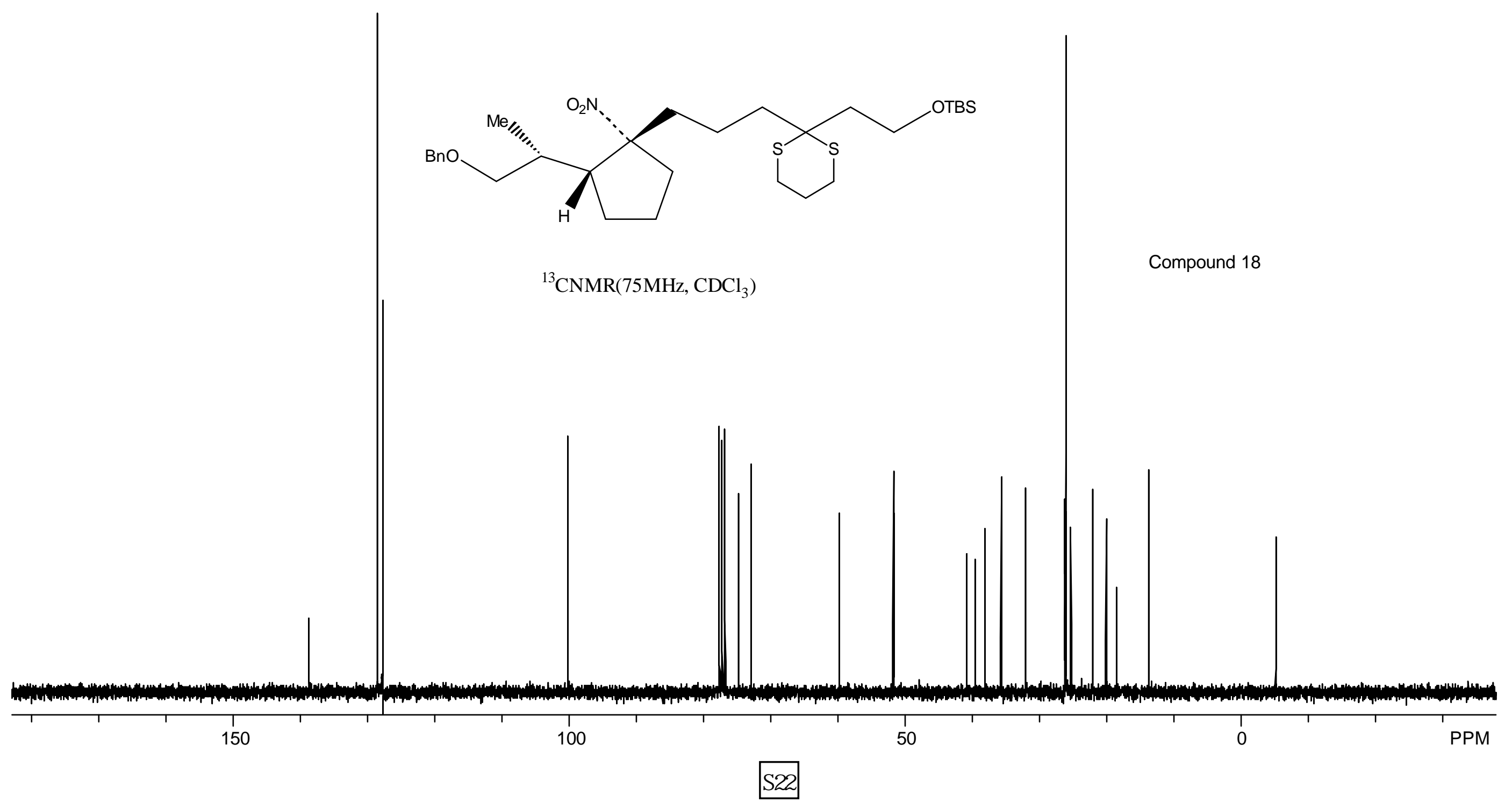



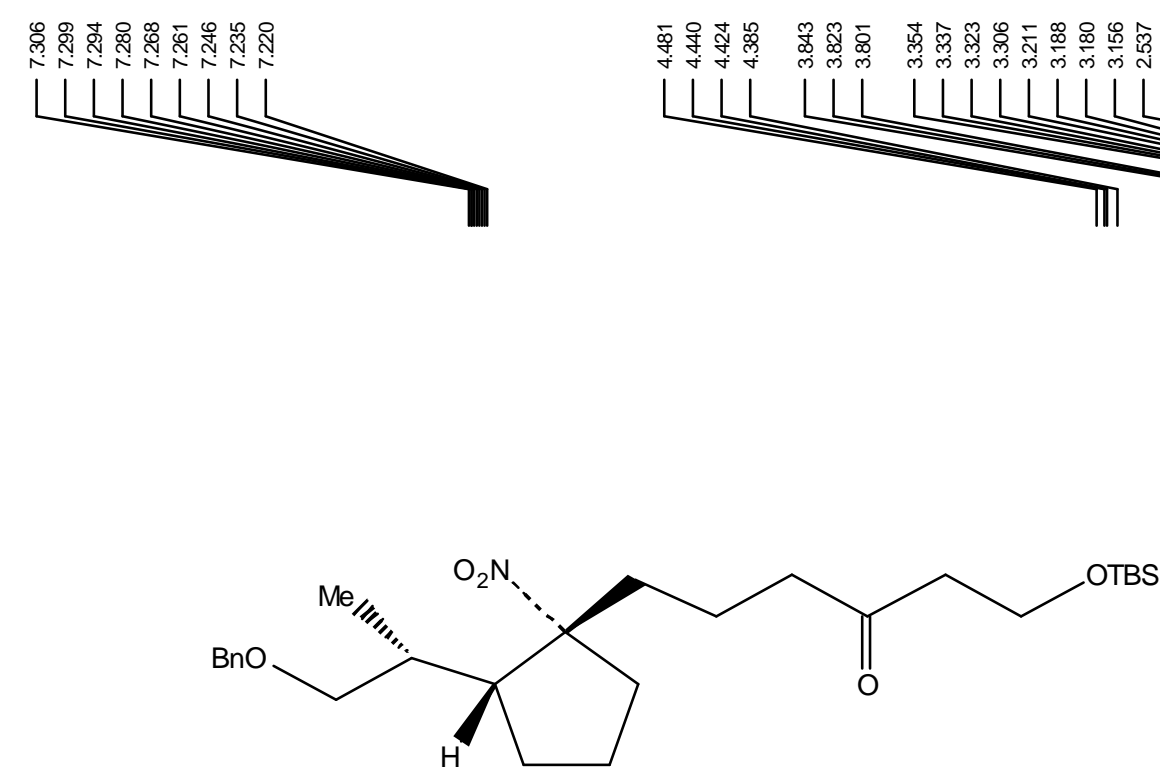

${ }^{1} \mathrm{HNMR}\left(300 \mathrm{MHz}, \mathrm{CDCl}_{3}\right)$

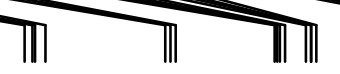

IIIIII Min

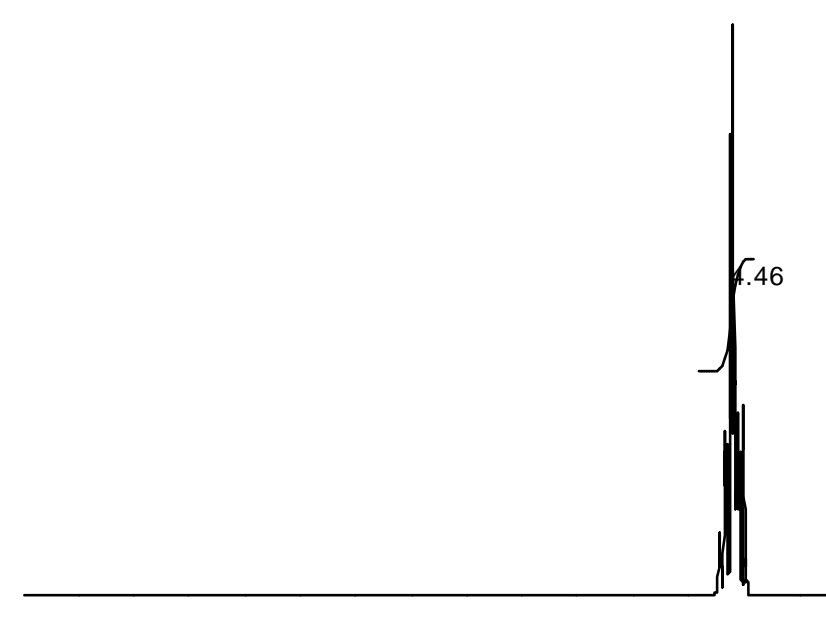

Compound 19 

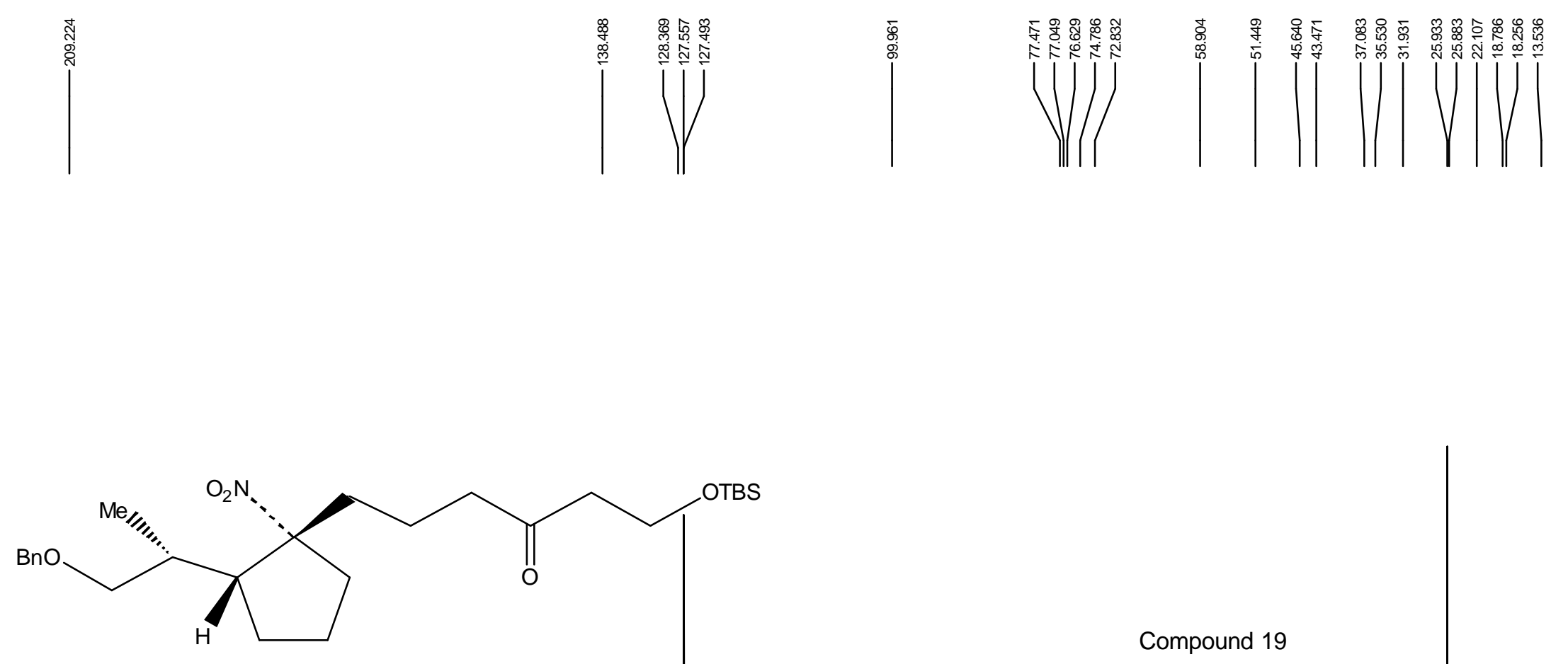

Compound 19

${ }^{13} \mathrm{CNMR}\left(75 \mathrm{MHz}, \mathrm{CDCl}_{3}\right)$

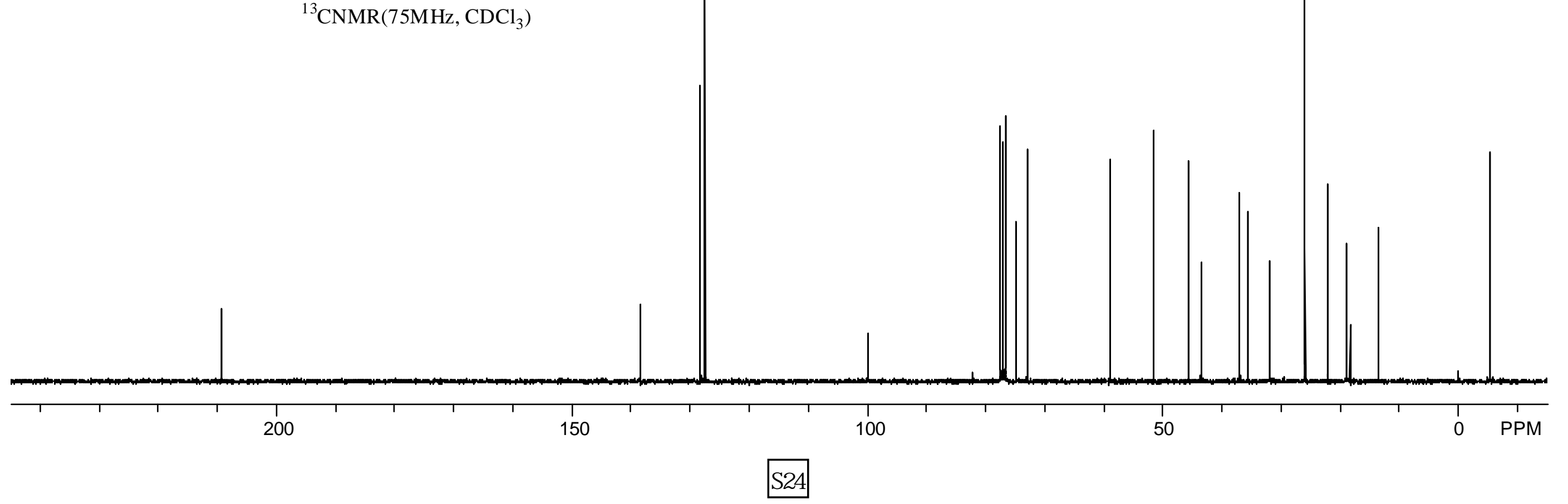




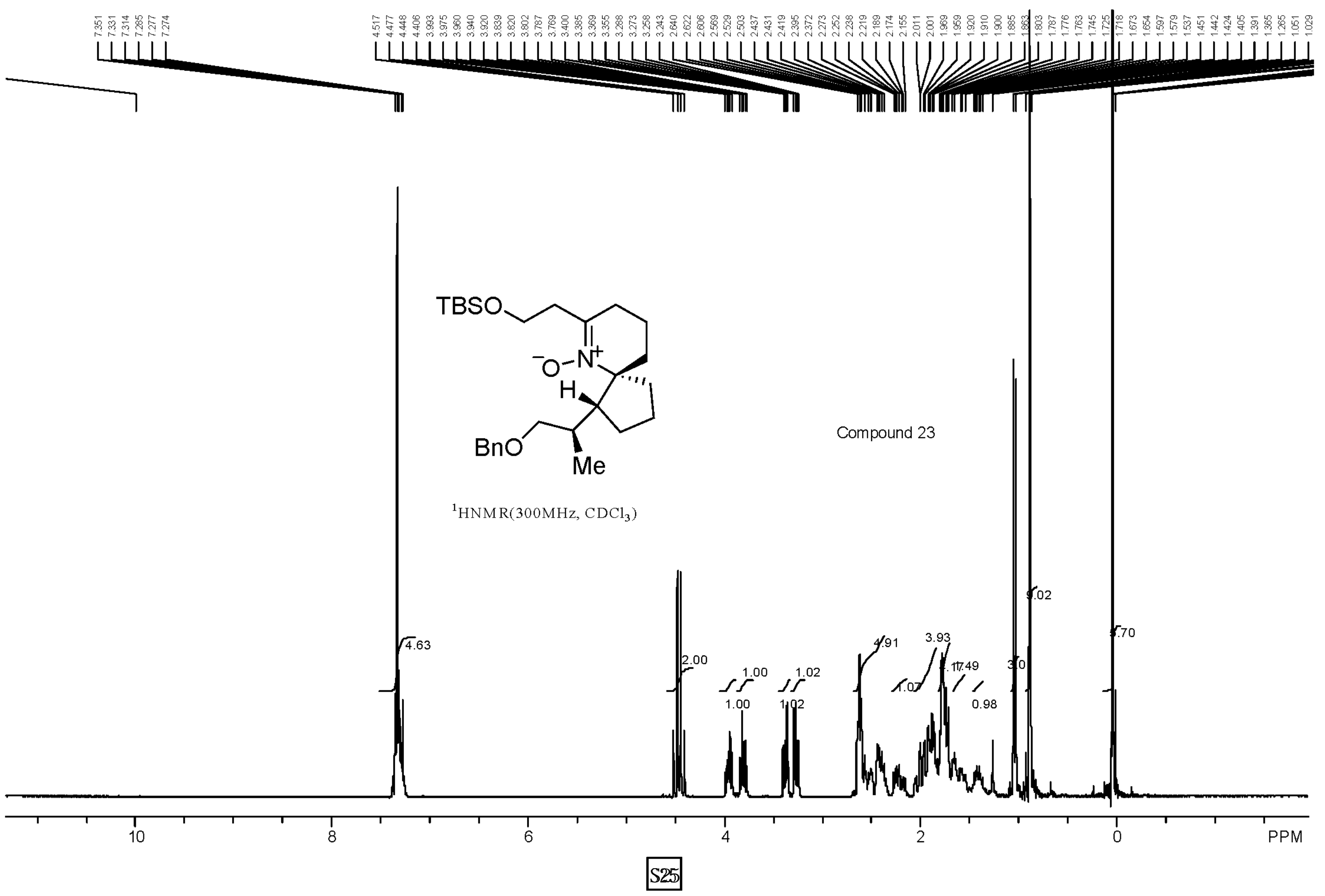




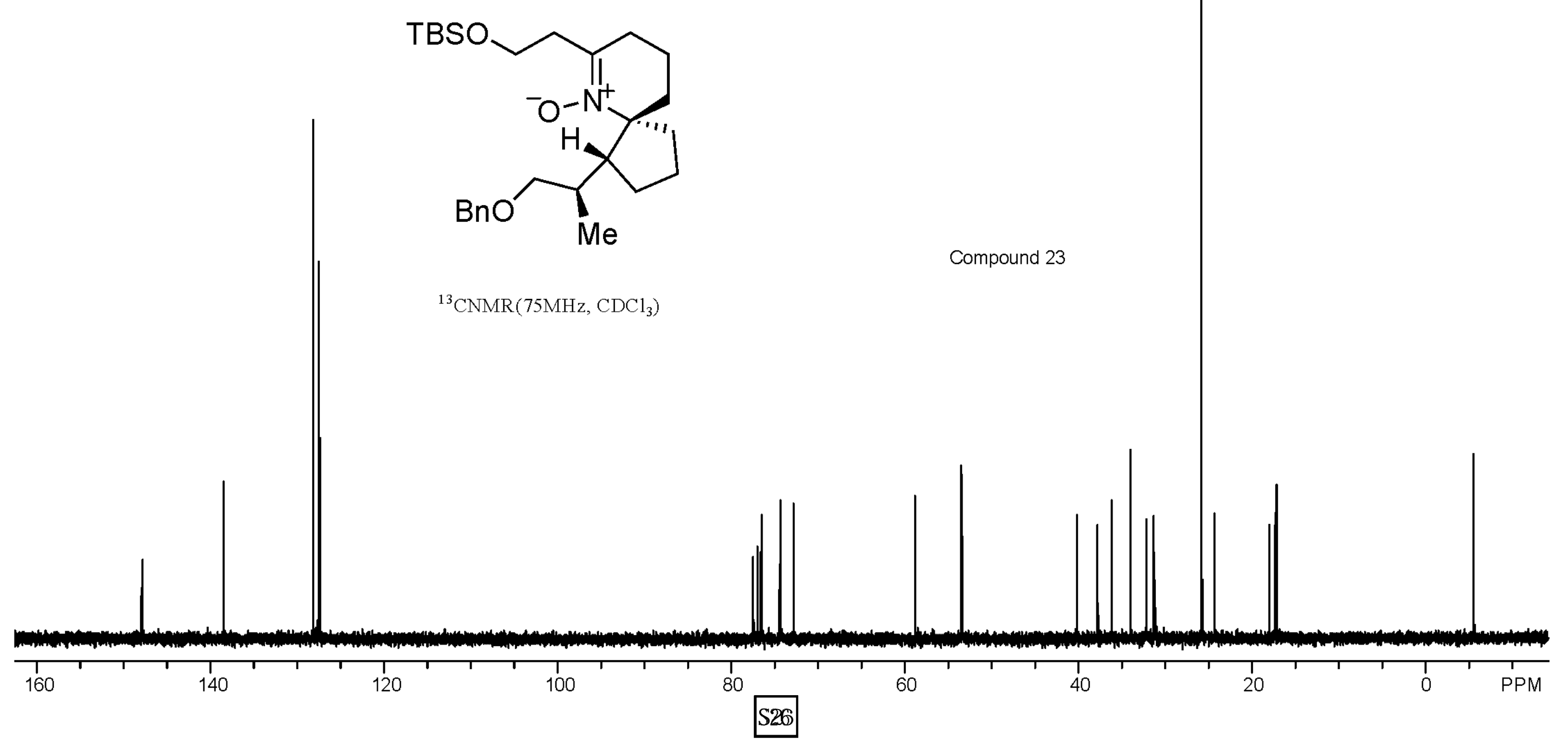



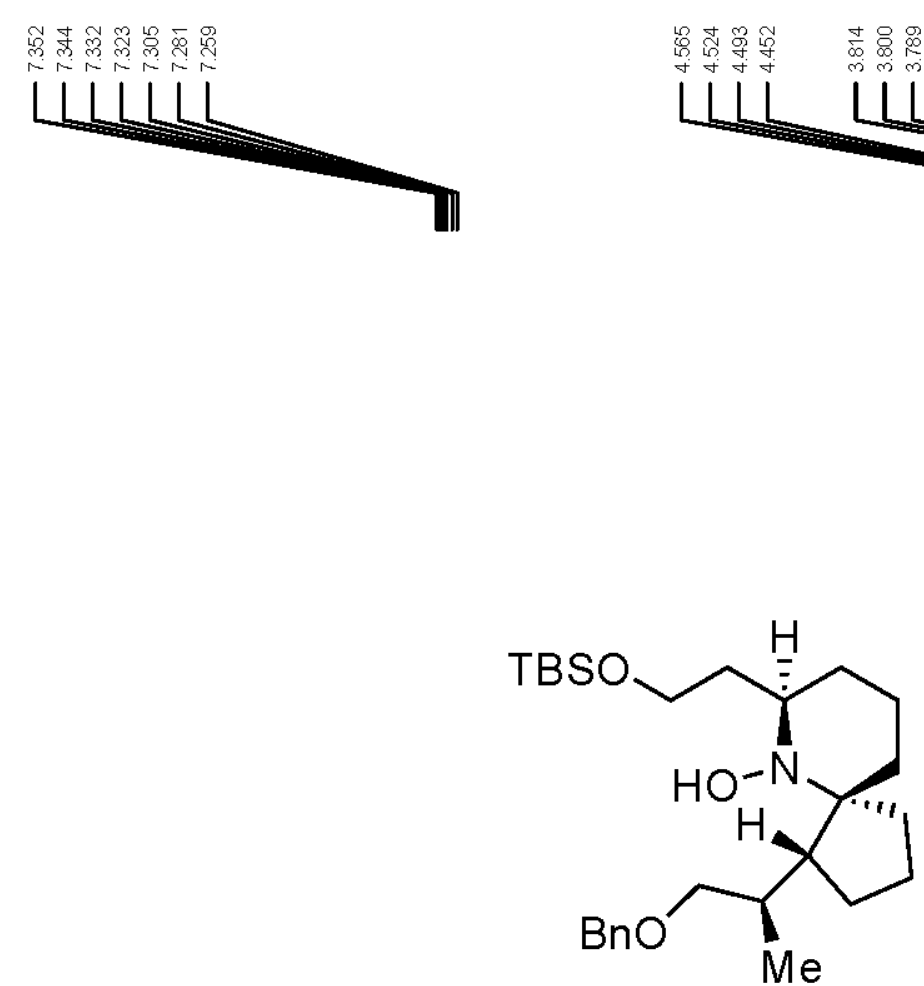

Compound 24

${ }^{1} \mathrm{HNMR}\left(300 \mathrm{MHz}, \mathrm{CDCl}_{3}\right)$
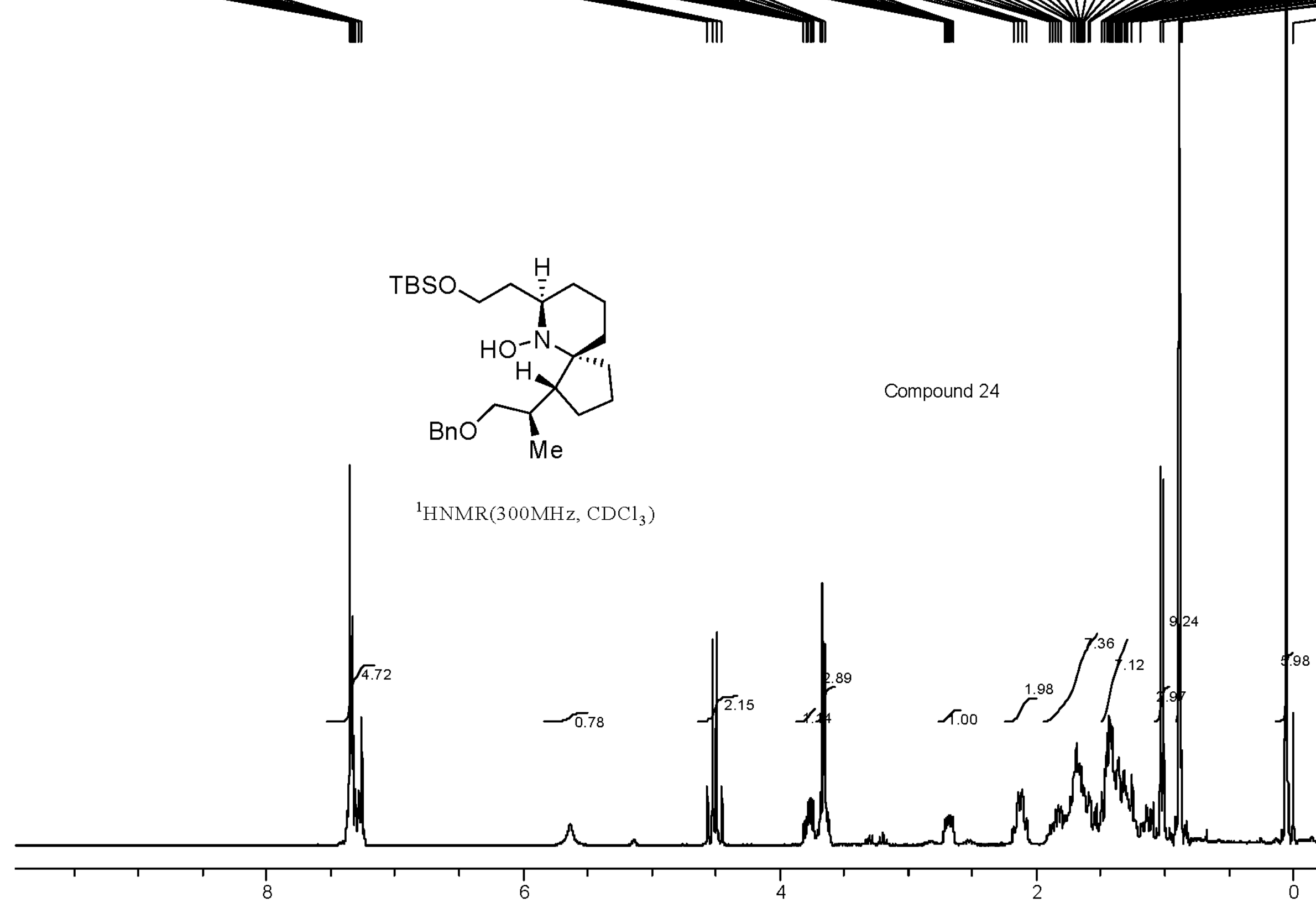


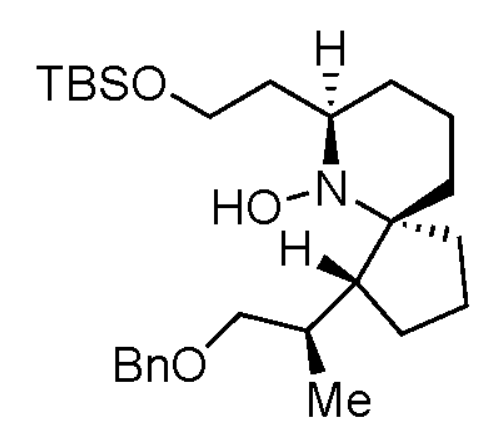

Compound 24

${ }^{13} \mathrm{CNMR}\left(75 \mathrm{MHz}, \mathrm{CDCl}_{3}\right.$ )

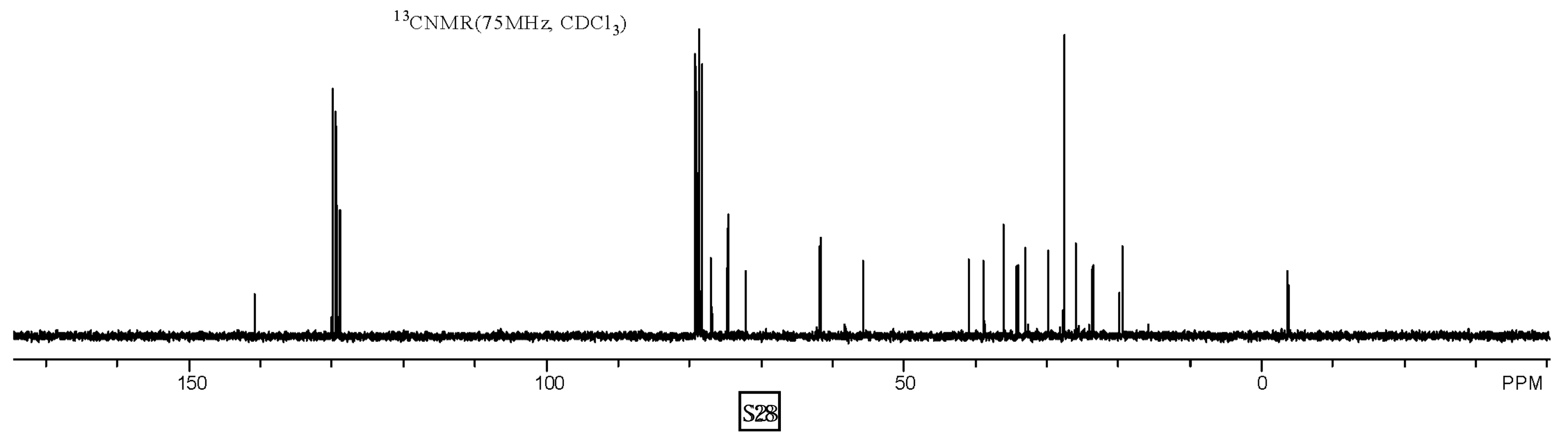




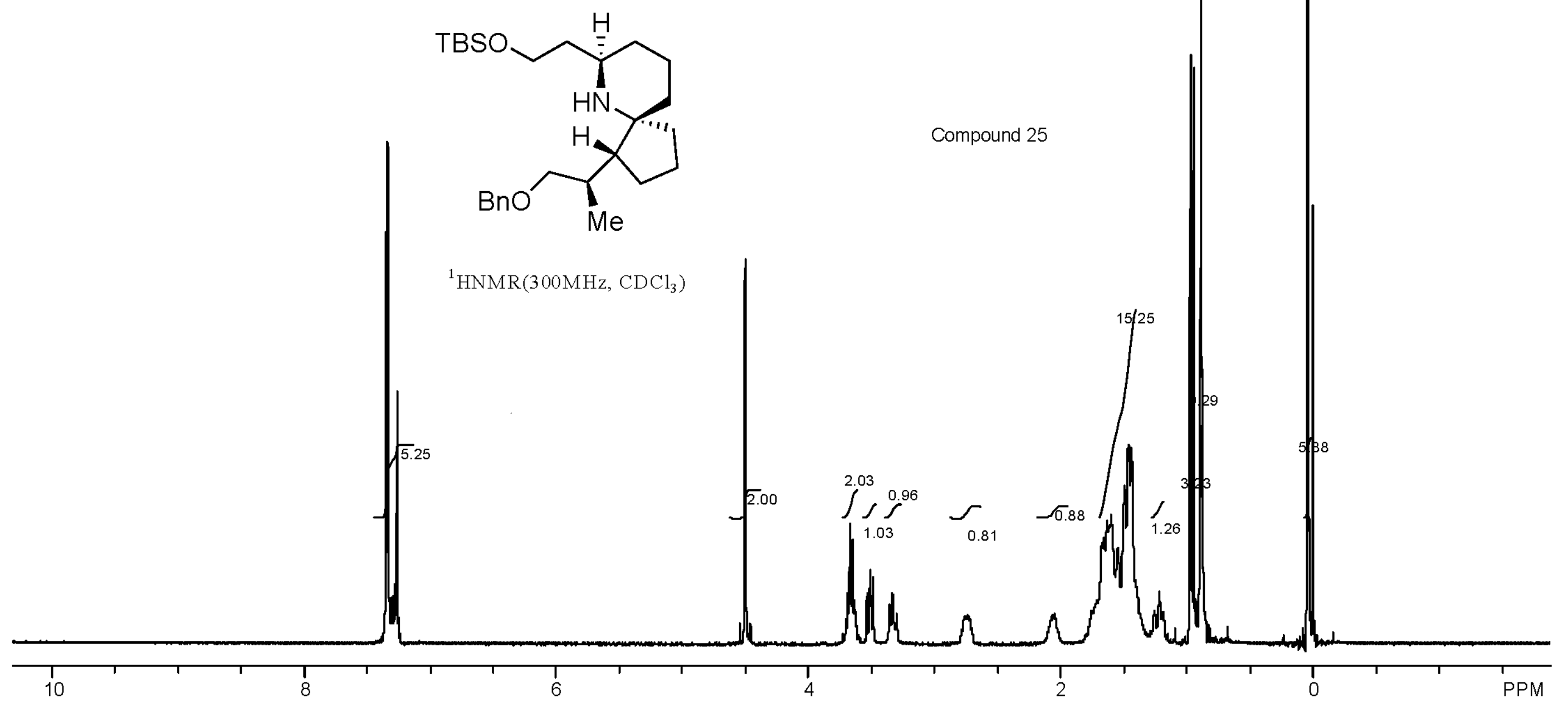

$\$ 29$ 

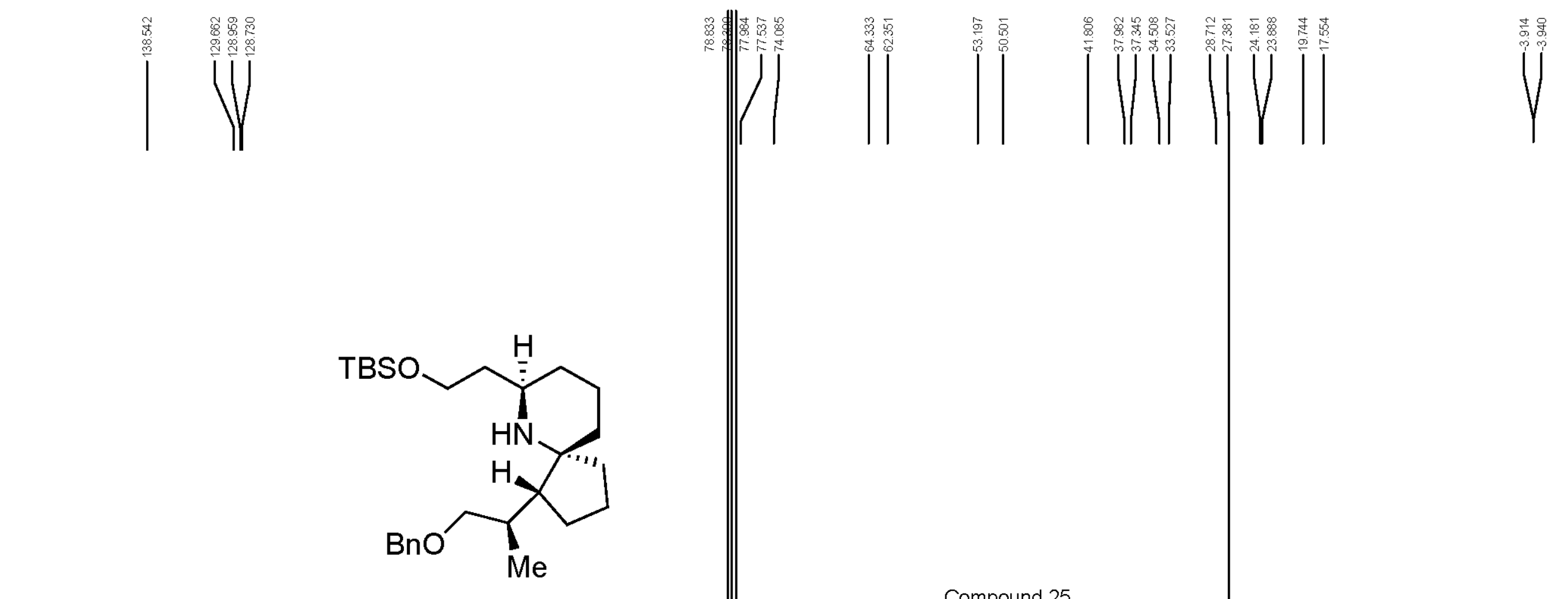

${ }^{13} \mathrm{CNMR}\left(75 \mathrm{MHz}, \mathrm{CDCl}_{3}\right)$

Compound 25
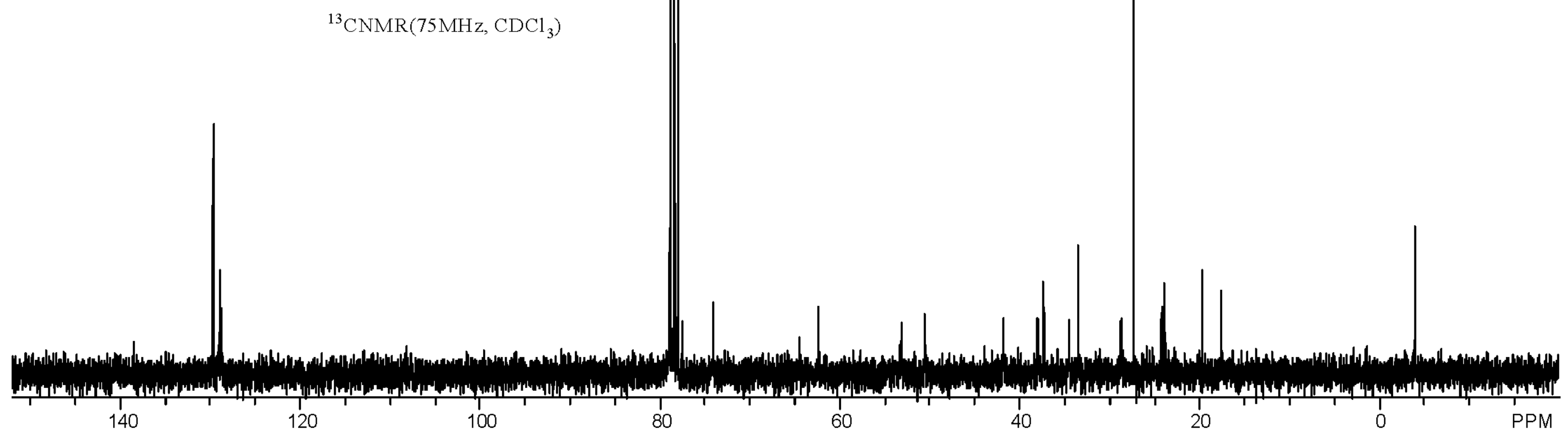


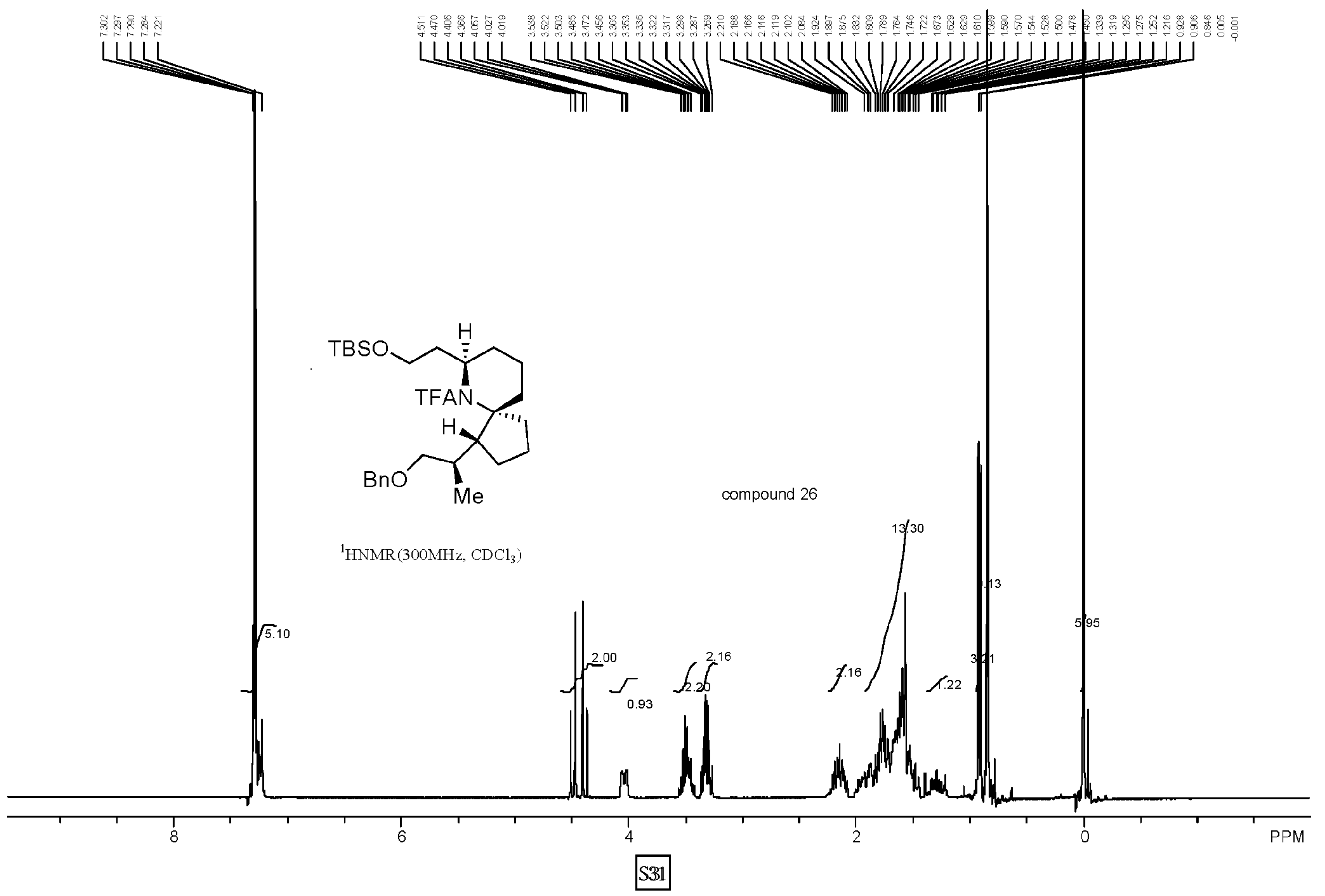



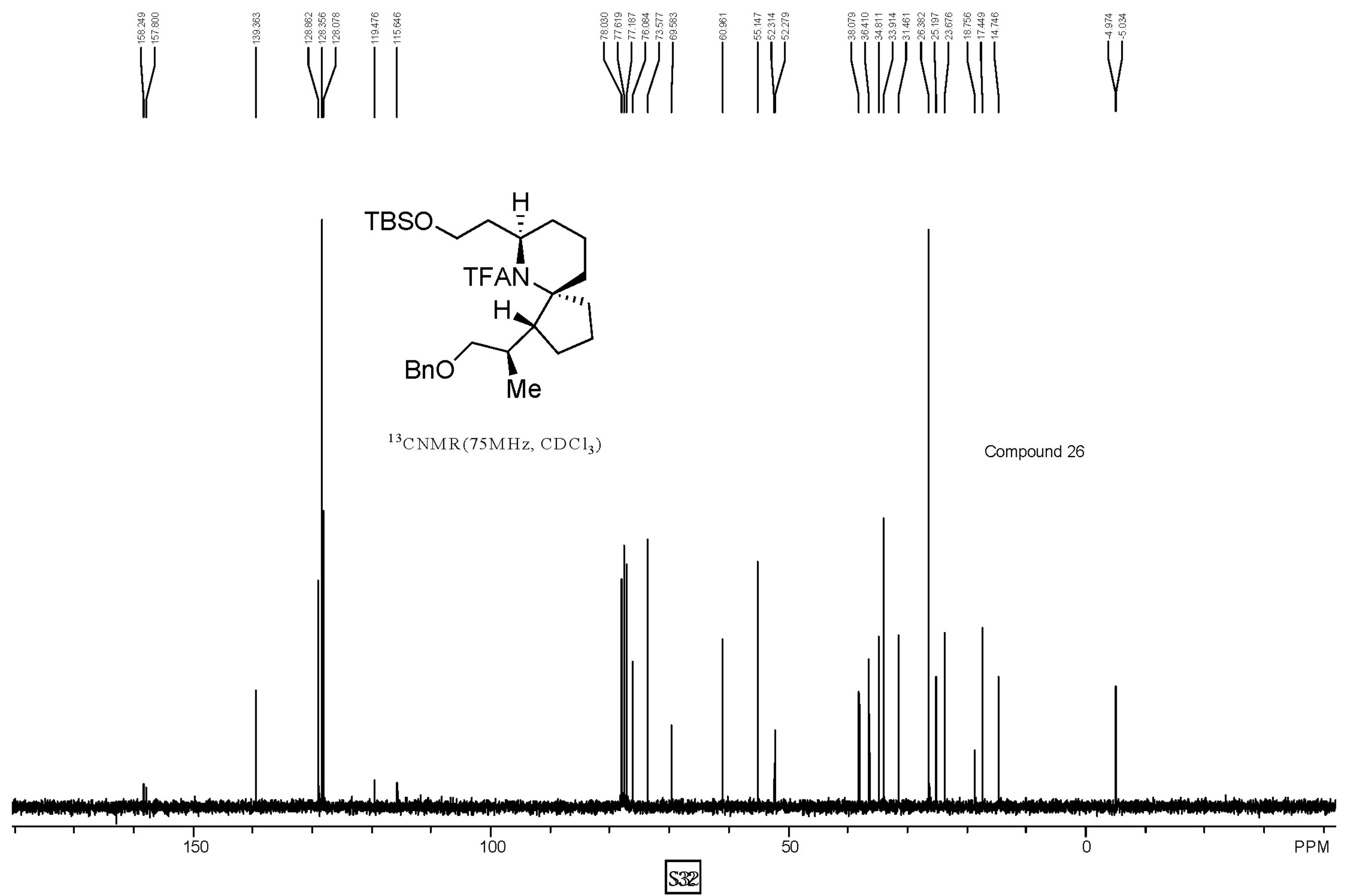


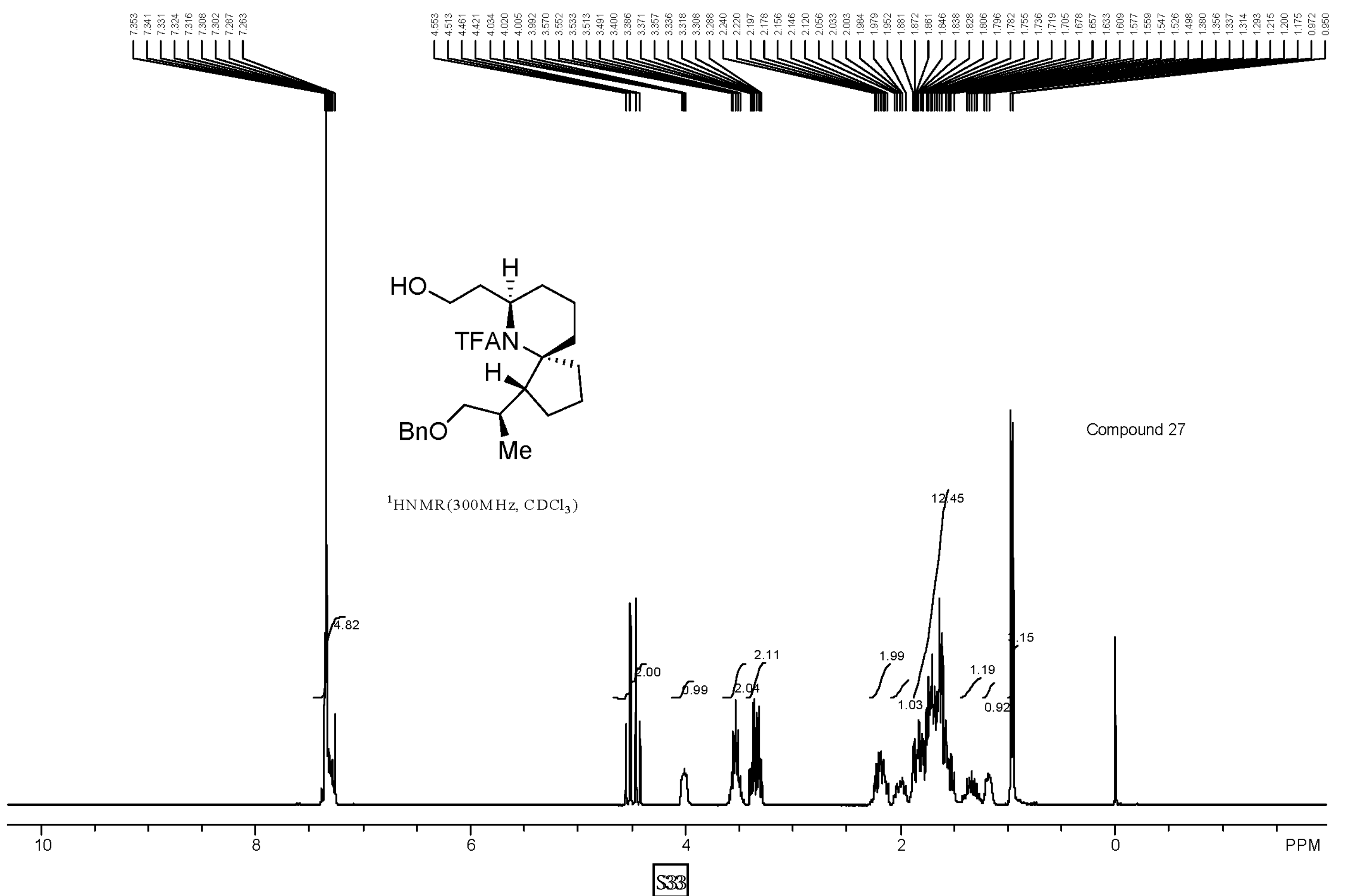




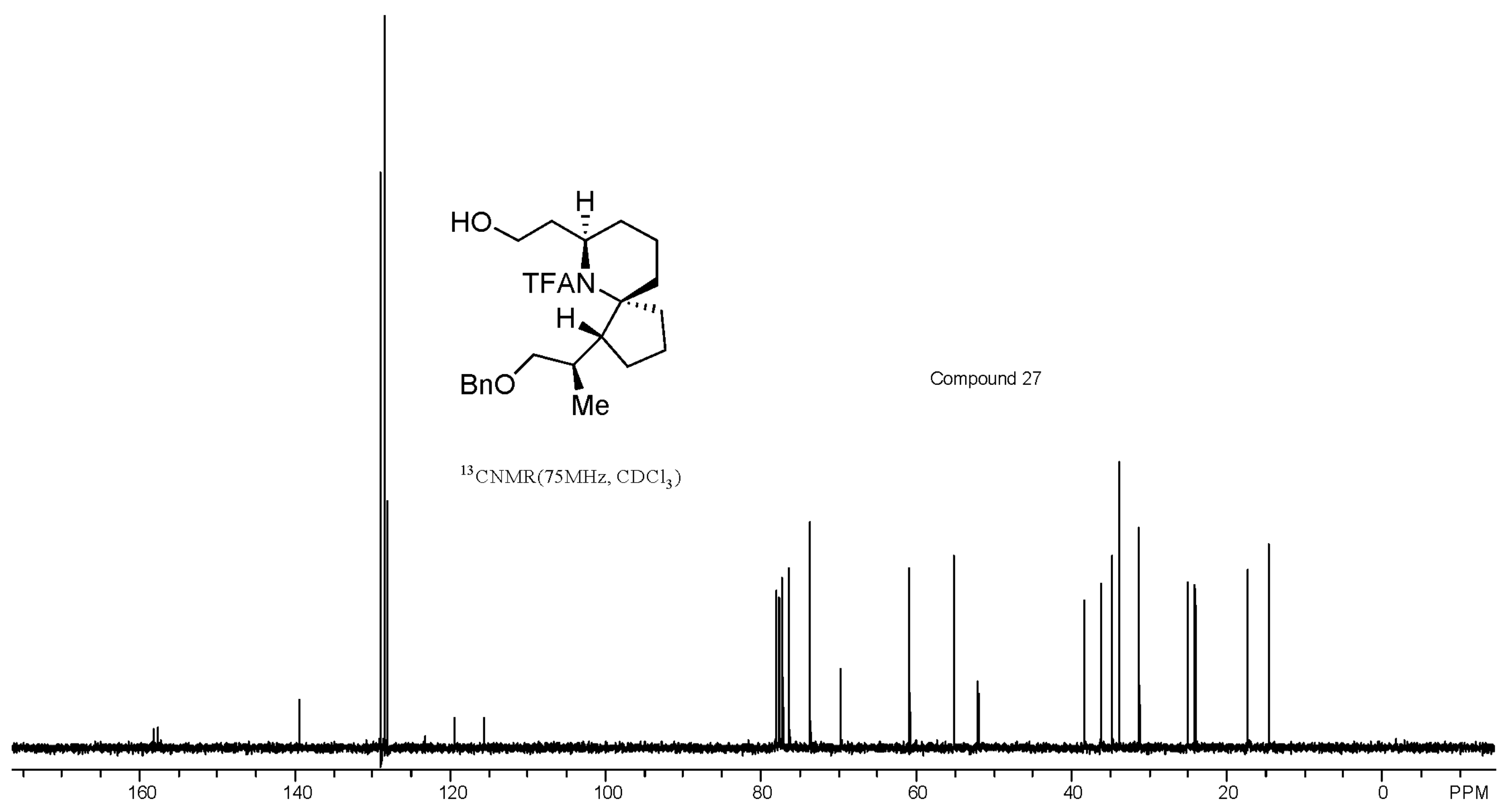

$\$ 34$ 


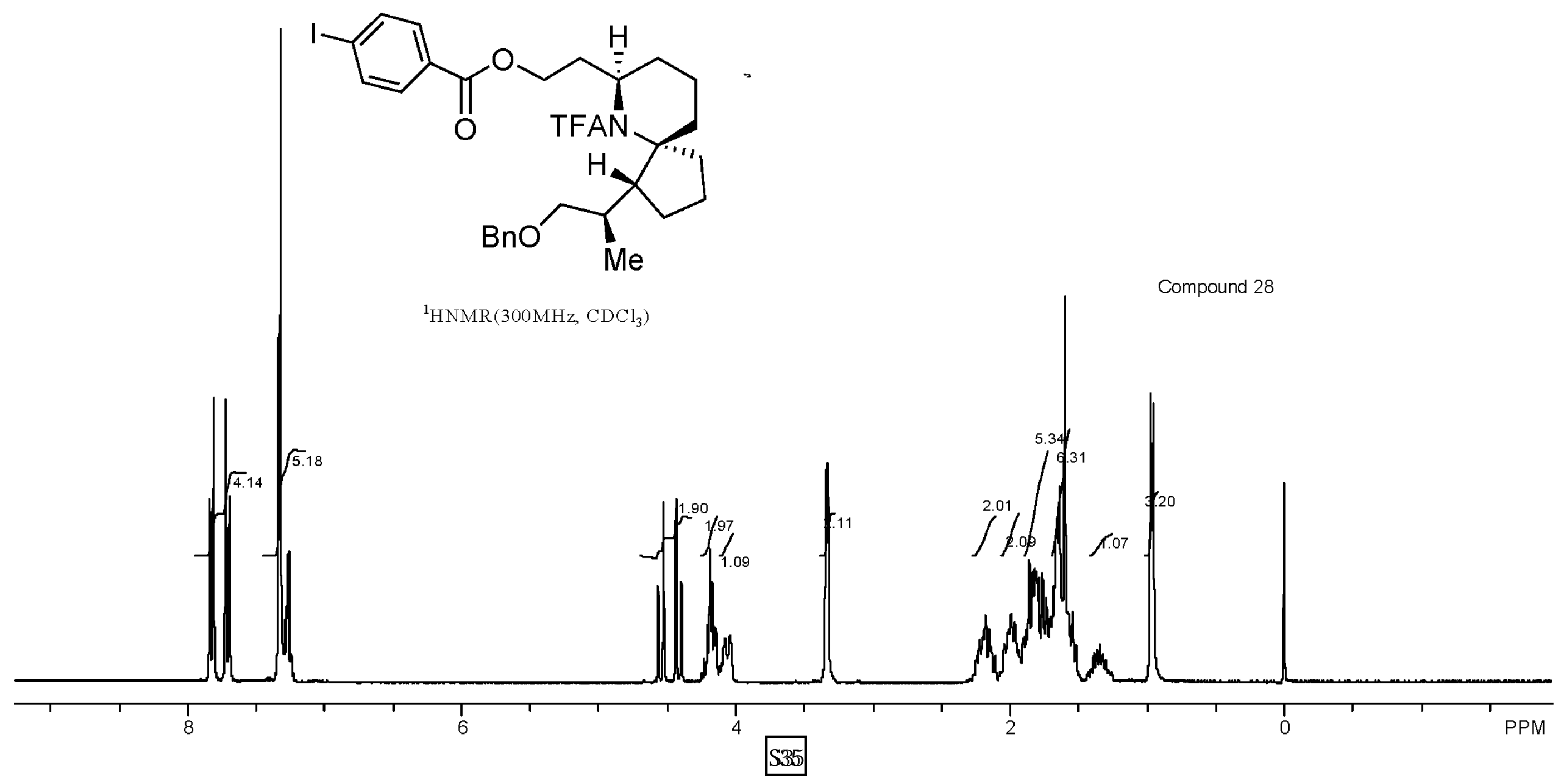



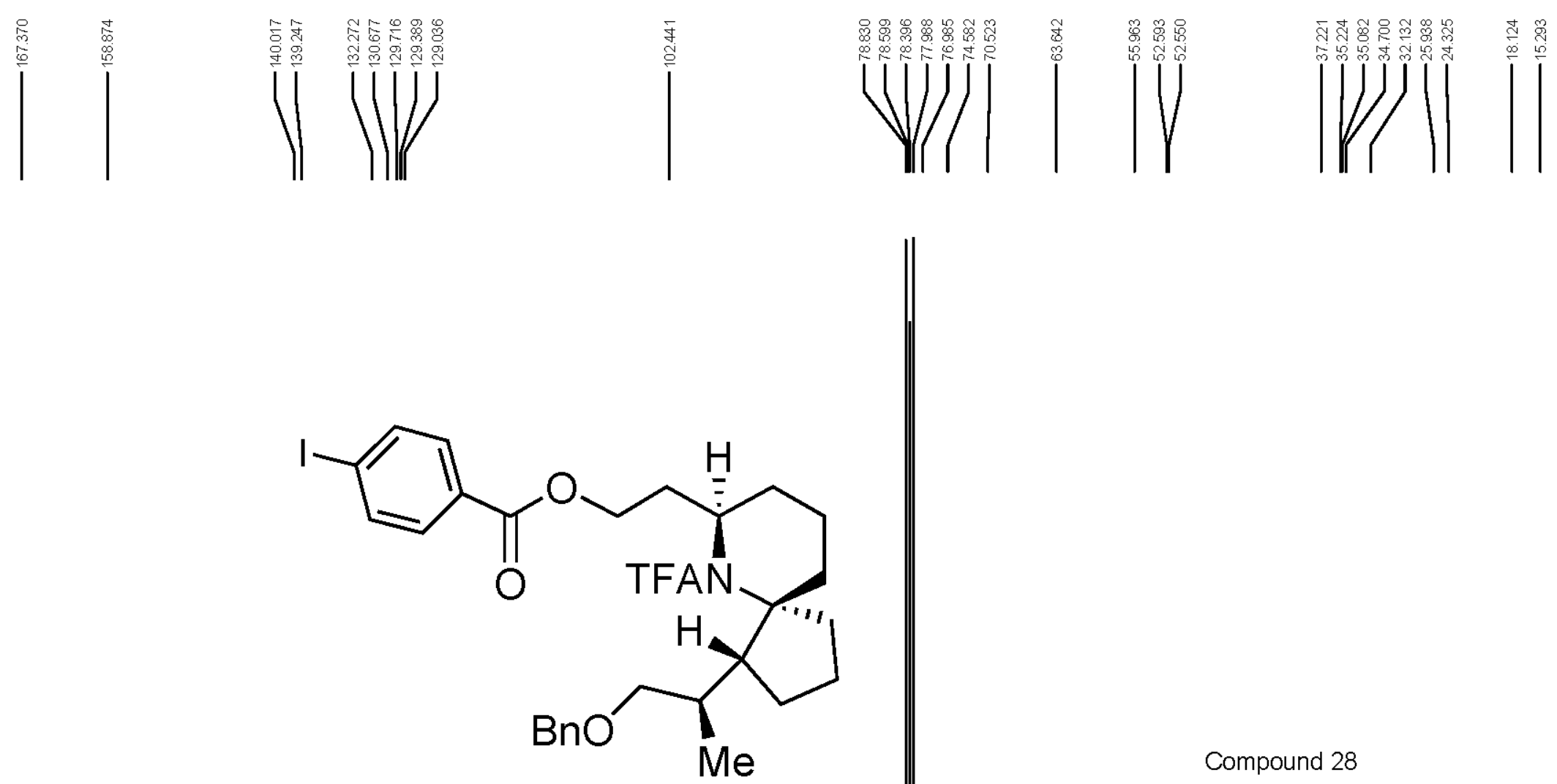

Compound 28

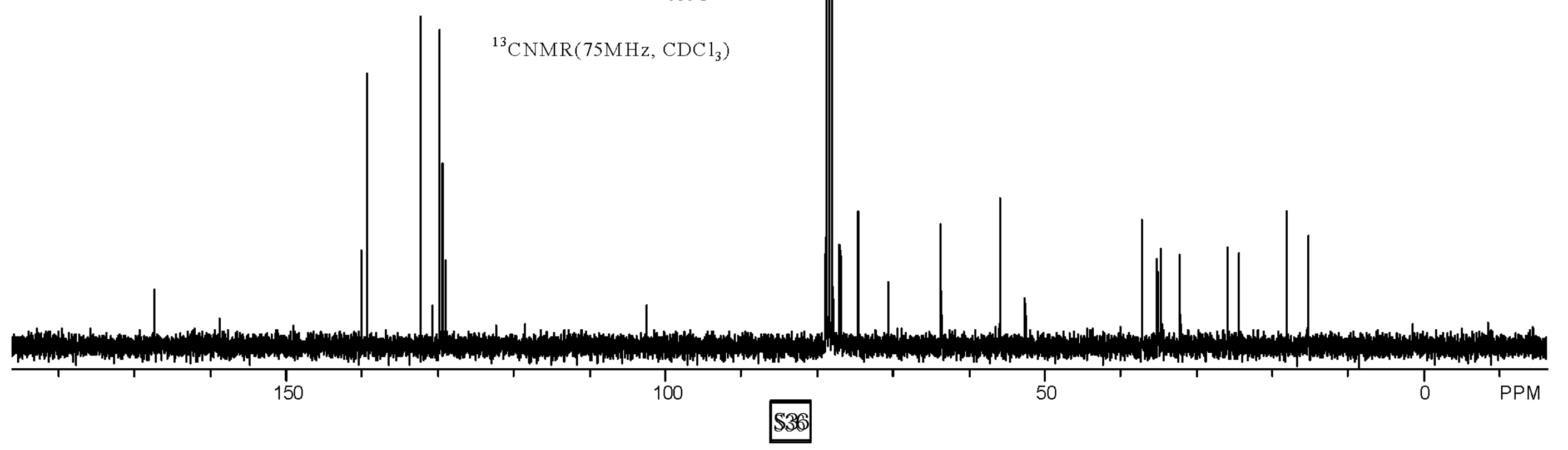




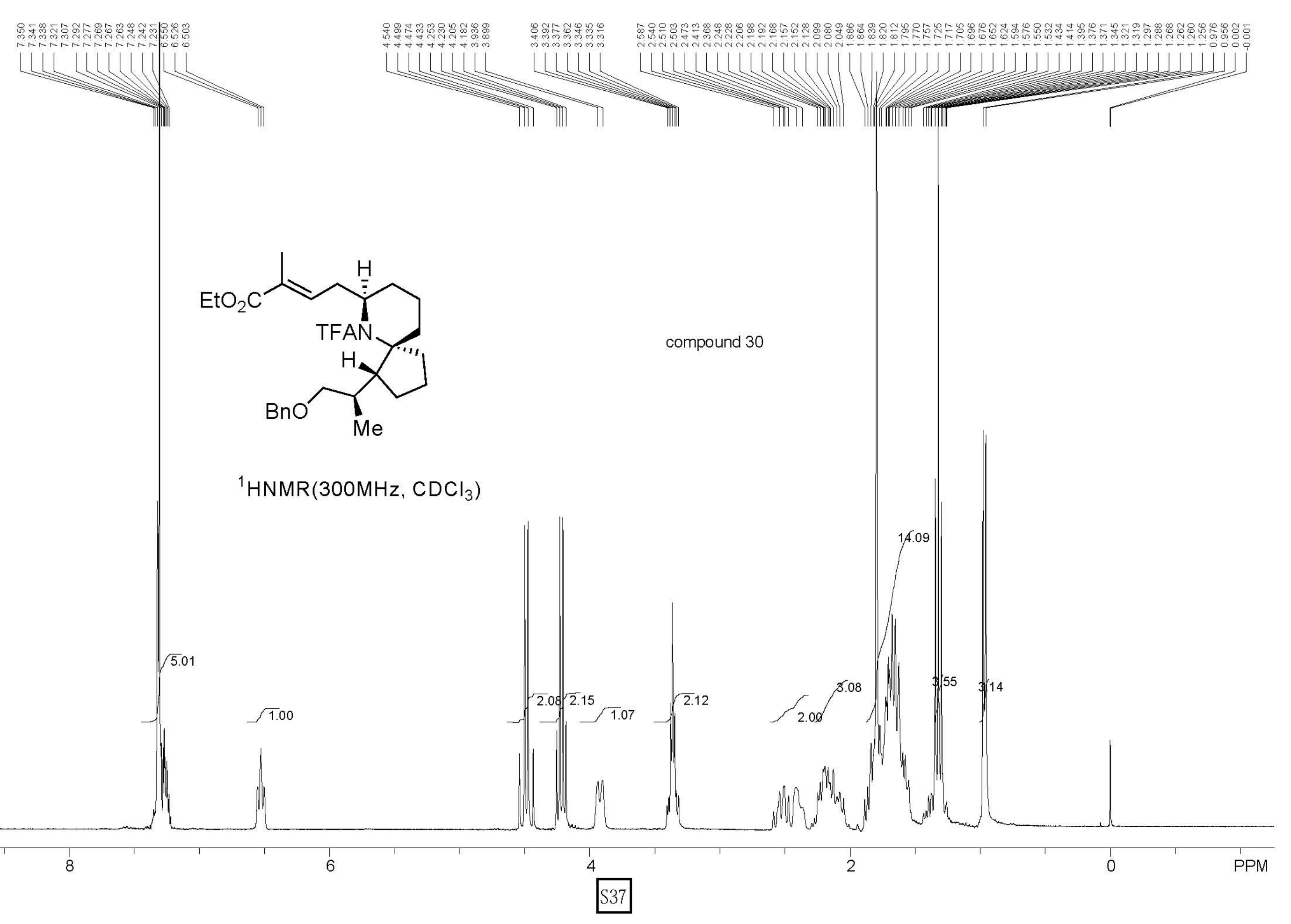




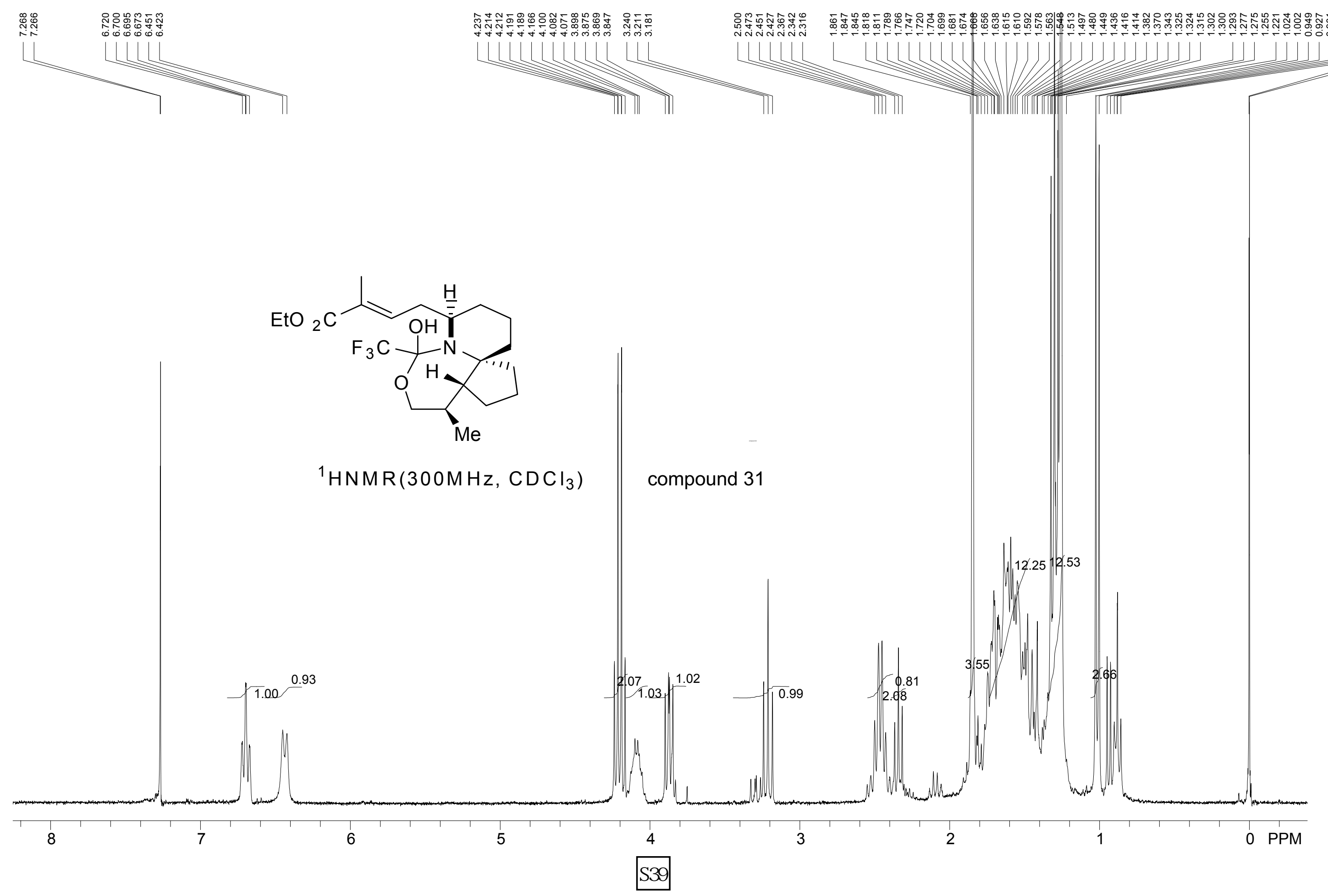




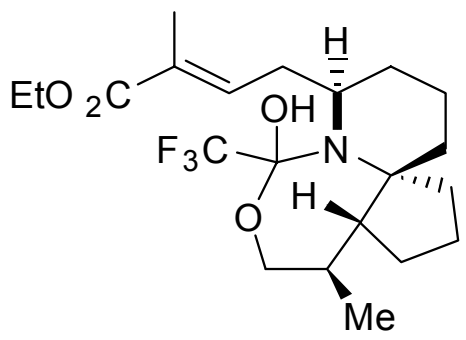

${ }^{13} \mathrm{CNMR}\left(75 \mathrm{MHz}, \mathrm{CDCl}_{3}\right)$

compound 31 


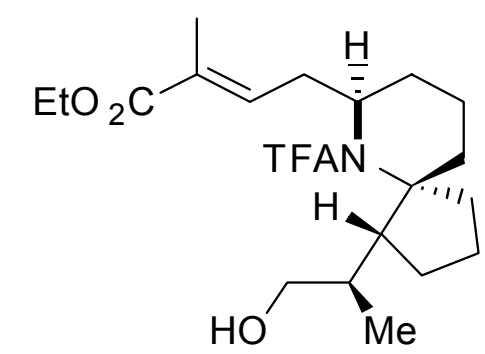

${ }^{1} \mathrm{HNMR}\left(300 \mathrm{MHz}, \mathrm{CDCl}_{3}\right)$

compound 3

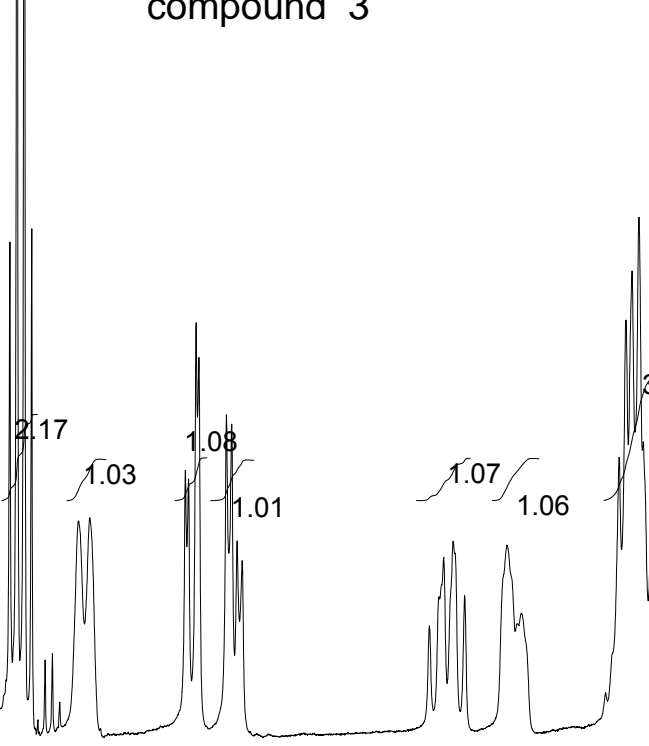

15.34

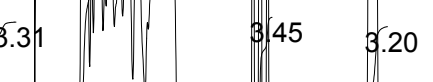

S41 


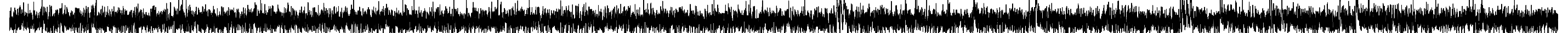

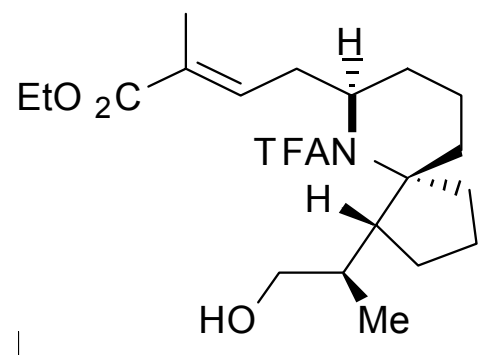

${ }^{13} \mathrm{CNMR}\left(75 \mathrm{MHz}, \mathrm{CDCI}_{3}\right)$ 

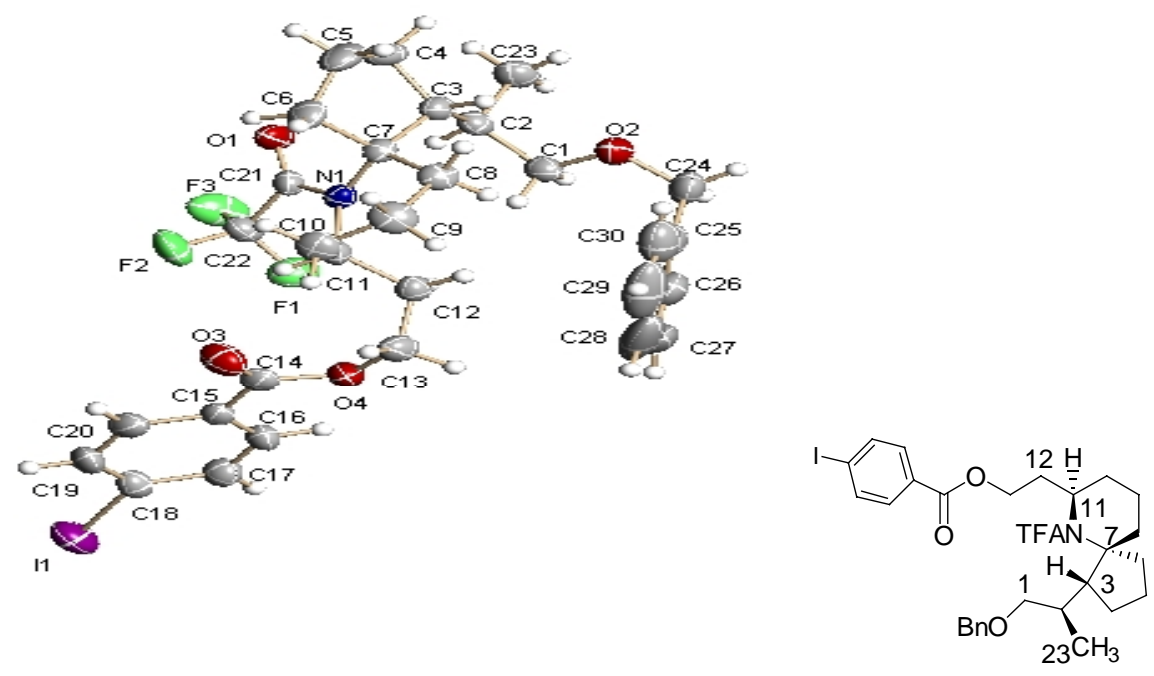

ORTEP drawing of the X-ray structure of $\mathbf{2 8}$ 